\title{
ON THE REDUCTIONS AND CLASSICAL SOLUTIONS OF THE SCHLESINGER EQUATIONS.
}

\author{
B. DUBROVIN AND M. MAZZOCCO
}

To the memory of our friend Andrei Bolibruch

\begin{abstract}
The Schlesinger equations $S_{(n, m)}$ describe monodromy preserving deformations of order $m$ Fuchsian systems with $n+1$ poles. They can be considered as a family of commuting time-dependent Hamiltonian systems on the direct product of $n$ copies of $m \times m$ matrix algebras equipped with the standard linear Poisson bracket. In this paper we address the problem of reduction of particular solutions of "more complicated" Schlesinger equations $S_{(n, m)}$ to "simpler" $S_{\left(n^{\prime}, m^{\prime}\right)}$ having $n^{\prime}<n$ or $m^{\prime}<m$.
\end{abstract}

\section{Contents}

1. Introduction.

2

2. Schlesinger equations as monodromv preserving deformations of Fuchsian svstems. 10

2.1. Levelt basis near a logarithmic singularitv and local monodromv data 11

2.2. Monodromv data and isomonodromic deformations of a Fuchsian svstem 14

3. Reductions of the Schlesinger svstems. 19

3.1. Reducible monodromv groups. 19

3.2. An example. 26

3.3. Smaller monodromv groups. 27

References 30

1991 Mathematics Subject Classification. 32G34 (Primary); 34M55, 53D30 (Secondary).

Key words and phrases. Monodromy preserving deformations.

The authors wish to dedicate this paper to the memory of A. Bolibruch who was a great source of inspiration. We are grateful to B. Malgrange, H. Umemura and M. Singer for many helpful conversations. The researches of B.D. were partially supported by Italian Ministry of Education research grant Cofin2004 "Geometry of Integrable Systems", and also by the ESF research grant MISGAM. The researches of M.M. was supported by EPSRC, SISSA, IRMA (Strasbourg) and the European network ENIGMA. Finally, the authors wish to thank the referee for carefully reading the manuscript and for her/his helpful comments. 


\section{INTRODUCTION.}

The Schlesinger equations $S_{(n, m)}$ [35] is the following system of nonlinear differential equations

$$
\begin{aligned}
\frac{\partial}{\partial u_{j}} A_{i} & =\frac{\left[A_{i}, A_{j}\right]}{u_{i}-u_{j}}, \quad i \neq j, \\
\frac{\partial}{\partial u_{i}} A_{i} & =-\sum_{j \neq i} \frac{\left[A_{i}, A_{j}\right]}{u_{i}-u_{j}},
\end{aligned}
$$

for $m \times m$ matrix valued functions $A_{1}=A_{1}(u), \ldots, A_{n}=A_{n}(u)$, where the independent variables $u=\left(u_{1}, \ldots, u_{n}\right)$ must be pairwise distinct. The first non-trivial case $S_{(3,2)}$ of the Schlesinger equations corresponds to the famous sixth Painlevé equation [9, 35, 10, the most general of all Painlevé equations. In the case of any number $n>3$ of $2 \times 2$ matrices $A_{j}$, the Schlesinger equations reduce to the Garnier systems $\mathcal{G}_{n}$ (see [10, 11, 32]).

The Schlesinger equations $S_{(n, m)}$ appeared in the theory of isomonodromic deformations of Fuchsian systems. Namely, the monodromy matrices of the Fuchsian system

$$
\frac{d \Phi}{d z}=\sum_{k=1}^{n} \frac{A_{k}(u)}{z-u_{k}} \Phi, \quad z \in \mathbb{C} \backslash\left\{u_{1}, \ldots, u_{n}\right\}
$$

do not depend on $u=\left(u_{1}, \ldots, u_{n}\right)$ if the matrices $A_{i}(u)$ satisfy (1.1). Conversely, under certain assumptions on the matrices $A_{1}, \ldots, A_{n}$ and for the matrix

$$
A_{\infty}:=-\left(A_{1}+\cdots+A_{n}\right),
$$

all isomonodromic deformations of the Fuchsian system are given by solutions to the Schlesinger equations (see, e.g., $[36)^{1}$.

The solutions to the Schlesinger equations can be parameterized by the monodromy data of the Fuchsian system (1.2) (see precise definition below in Section 2). To reconstruct the solution starting from given monodromy data one is to solve the classical Riemann - Hilbert problem of reconstruction of the Fuchsian system from its monodromy data. The main outcome of this approach says that the solutions $A_{i}(u)$ can be continued analytically to meromorphic functions on the universal covering of

$$
\left\{\left(u_{1}, \ldots, u_{n}\right) \in \mathbb{C}^{n} \mid u_{i} \neq u_{j} \text { for } i \neq j\right\}
$$

[24, 30]. This is a generalization of the celebrated Painlevé property of absence of movable critical singularities (see details in [14, 15]). In certain cases the technique based on the theory of Riemann-Hilbert problem gives a possibility

\footnotetext{
${ }^{1}$ Bolibruch constructed non-Schlesinger isomonodromic deformations in 4]. These can occur when the matrices $A_{i}$ are resonant, i.e. admit pairs of eigenvalues with positive integer differences. To avoid such non-Schlesinger isomonodromic deformations, we need to extend the set of monodromy data (see Section 2 below).
} 
to compute the asymptotic behavior of the solutions to the Schlesinger equations near the critical locus $u_{i}=u_{j}$ for some $i \neq j$, although, in general, the problem of determining the asymptotic behaviuor near the critical points is still open [17, 7, 12, 5].

It is the Painlevé property that was used by Painlevé and Gambier as the basis for their classification scheme of nonlinear differential equations. Of the list of some 50 second order nonlinear differential equations possessing Painlevé property the six (nowadays known as Painlevé equations) are selected due to the following crucial property: the general solutions to these six equations cannot be expressed in terms of classical functions, i.e., elementary functions, elliptic and other classical transcendental functions (see 37 for a modern approach to this theory based on a nonlinear version of the differential Galois theory). In particular, according to these results the general solution to the Schlesinger system $S_{(3,2)}$ corresponding to Painlevé-VI equation cannot be expressed in terms of classical functions.

A closely related question is the problem of construction and classification of classical solutions to Painlevé equations and their generalizations. This problem remains open even for the case of Painlevé-VI although there are interesting results based on the theory of symmetries of Painlevé equations 34, 33,1 and on the geometric approach to studying the space of monodromy data [7, 13, 27, 28,

The above methods do not give any clue to solution of the following general problems: are solutions of $S_{(n+1, m)}$ or of $S_{(n, m+1)}$ more complicated than those of $S_{(n, m)}$ ? Which solutions to $S_{(n+1, m)}$ or $S_{(n, m+1)}$ can be expressed via solutions to $S_{(n, m)}$ ? Furthermore, which of them can ultimately be expressed via classical functions?

In this paper we aim to suggest a general approach to the theory of reductions and classical solutions of the general Schlesinger equations $S_{(n, m)}$ for all $n, m$, based on the Riemann-Hilbert problem and on the group-theoretic properties of the monodromy group of the linear system (1.2). Our approach consists in determining the monodromy data of the Fuchsian system (1.2) that guarantee to have a reduction to $S_{(n-1, m)}$ or $S_{(n, m-1)}$ and eventually a classical solution.

We need a few definitions. Let us fix a solution to the Schlesinger equations $S_{(n, m)}$. Applying the algebraic operations and differentiations to the matrix entries of this solution we obtain a field $\mathcal{S}_{(n, m)}$ equipped with $n$ pairwise commuting differentiations $\partial / \partial u_{1}, \ldots, \partial / \partial u_{n}$, to be short a differential field. Define the rational closure $\mathcal{K}$ of a differential field $\mathcal{S}$ represented by functions of $n$ variables by taking rational functions with coefficients in $\mathcal{S}$

$$
\mathcal{K}:=\mathcal{S}\left(u_{1}, \ldots, u_{n}\right)
$$


Taking the rational closure of the differential field $S_{(n, m)}$, we obtain the differential field $\mathcal{K}_{(n, m)}$. (Needless to say that the field $\mathcal{K}_{(n, m)}$ depends on the choice of the solution to the Schlesinger equations $S_{(n, m)}$.)

We now construct new differential fields obtained from $\mathcal{K}_{\left(n_{1}, m_{1}\right)}, \ldots, \mathcal{K}_{\left(n_{k}, m_{k}\right)}$ by applying one or more of the following admissible elementary operations.

1. Tensor product. Given two differential fields $\mathcal{K}_{1}$ and $\mathcal{K}_{2}$ represented by functions of $n_{1}$ and $n_{2}$ variables $u_{1}, \ldots, u_{n_{1}}$ and $v_{1}, \ldots, v_{n_{2}}$ respectively, produce new differential field $\mathcal{K}_{1} \otimes \mathcal{K}_{2}$ taking the rational closure of the minimal differential field of functions of $n_{1}+n_{2}$ independent variables $u_{1}, \ldots, u_{n_{1}}, v_{1}$, $\ldots, v_{n_{2}}$ containing both $K_{1}$ and $K_{2}$. A particular case of this operation is

2. Addition of an independent variable. Given a differential field $\mathcal{K}$ represented by functions of $n$ variables $u_{1}, \ldots, u_{n}$ define an extension $\tilde{\mathcal{K}} \supset \mathcal{K}$ by taking rational functions of a new independent variable $u_{n+1}$ with coefficients in $\mathcal{K}$,

$$
\tilde{\mathcal{K}}=\mathcal{K} \otimes \mathbb{C}\left(u_{n+1}\right) .
$$

3. Given two differential fields $\mathcal{K}_{1}, \mathcal{K}_{2}$ represented by functions of the same number of variables $n$, define the composite $\mathcal{K}_{1} \mathcal{K}_{2}$ taking the minimal differential field of functions of $n$ variables containing both $\mathcal{K}_{1}$ and $\mathcal{K}_{2}$ and applying the rational closure procedure.

4. A differential field extension $\mathcal{K}^{\prime} \supset \mathcal{K}$ is said to be of the Picard-Vessiot type if it is the minimal rationally closed differential field of functions of $n$ variables containing solutions of a Pfaffian linear system with coefficients in $\mathcal{K}$ 20, 38.

Recall that a Pfaffian linear system of the order $k$ with coefficients in a differential field $\mathcal{K}$ represented by functions of $n$ variables reads

$$
\frac{\partial Y}{\partial u_{i}}=B_{i} Y, \quad i=1, \ldots, n, \quad Y=\left(y_{1}, \ldots, y_{k}\right)^{T}
$$

where the $n$ matrices

$$
B_{i} \in \operatorname{Mat}(k ; \mathcal{K}) \text { for } i=1, \ldots, n
$$

must satisfy

$$
\frac{\partial B_{i}}{\partial u_{j}}-\frac{\partial B_{j}}{\partial u_{i}}+\left[B_{i}, B_{j}\right]=0, \quad \text { for all } i \neq j .
$$

The linear space of solutions of the Pfaffian system is finite dimensional. The differential field $\mathcal{K}^{\prime}$ is the minimal extension of $\mathcal{K}$ containing all components $y_{1}, \ldots, y_{k}$ of any of these solutions.

We will also denote $\mathcal{K}^{(N)}$ the differential field extension of $\mathcal{K}$ obtained by $N$ Picard-Vessiot type extensions of $\mathcal{K}$

$$
\mathcal{K} \subset \mathcal{K}^{\prime} \subset \mathcal{K}^{\prime \prime} \subset \cdots \subset \mathcal{K}^{(N)} .
$$


Using the above admissible extensions we can describe in what circumstances a particular solution to the Schlesinger equations $S_{(n, m)}$ can be expressed via solutions to $S_{\left(n^{\prime}, m^{\prime}\right)}$ with smaller $n^{\prime}$ or $m^{\prime}$. Similar results were obtained in 29] for the special case of $m=2$.

Theorem 1.1. Consider a solution to $S_{(n, m)}$ such that the eigenvalues of the matrix $A_{\infty}$ are pairwise distinct and the monodromy group of the associated Fuchsian system (1.2) admits a $k$-dimensional invariant subspace, $k>0$. Then this solution belongs to a Picard-Vessiot type extension $\mathcal{K}^{(N)}$ for some $N$ of the composite

$$
\mathcal{K}=\mathcal{K}_{(n, k)} \mathcal{K}_{(n, m-k)}
$$

where $\mathcal{K}_{(n, k)}$ and $\mathcal{K}_{(n, m-k)}$ are two differential fields associated with certain two solutions of the systems $\mathcal{S}_{(n, k)}$ and $\mathcal{S}_{(n, m-k)}$ respectively.

In particular,

Corollary 1.2. Given a solution to $S_{(n, m)}$ such that the monodromy group of the associated Fuchsian system 1.2) is upper-triangular and the eigenvalues of $A_{\infty}$ are pairwise distinct, it belongs to a Picard-Vessiot type extension $\mathcal{K}_{0}^{(N)}$ for some $N$ of

$$
\mathcal{K}_{0}=\mathbb{C}\left(u_{1}, \ldots, u_{n}\right) .
$$

The proof of this Theorem is based on the following two lemmata.

Lemma 1.3. Given a solution

$$
A(z ; u)=\sum_{i=1}^{n} \frac{A_{i}(u)}{z-u_{i}}
$$

to the Schlesinger equations $\mathcal{S}_{(n, m)}$ with diagonalizable matrix $A_{\infty}$ such that the associated monodromy representation has a $k$-dimensional invariant subspace, denote $\mathcal{K}_{(n, m)}$ the corresponding differential field. Then there exists a matrix

$$
G(z ; u) \in \overline{\mathcal{K}}_{(n, m)}[z], \quad \operatorname{det} G(z) \equiv 1
$$

such that all matrices $B_{i}(u), i=1, \ldots, n$ of the gauge equivalent Fuchsian system with

$$
B(z ; u)=G^{-1}(z ; u) A(z ; u) G(z ; u)+G^{-1}(z ; u) \frac{d G(z ; u)}{d z}=\sum_{i=1}^{n} \frac{B_{i}(u)}{z-u_{i}}
$$

have a u-independent $k$-dimensional common invariant subspace. Here $\overline{\mathcal{K}}_{(n, m)}$ is a Picard - Vessiot type extension of the field $\mathcal{K}_{(n, m)}$. Moreover, the matrices $B_{1}(u), \ldots, B_{n}(u)$ satisfy Schlesinger equations.

This lemma, apart from polynomiality of the gauge transformation in $z$, is the main result of the papers 22, 23 by S.Malek ${ }^{2}$. We give here a new short

\footnotetext{
${ }^{2}$ Actually, there is a stronger claim in the main result of 23 , namely, it is said that the coefficients of the reducing gauge transformation are rational functions in $u_{1}, \ldots, u_{n}$ and entries of $A_{1}(u), \ldots, A_{n}(u)$. We were unable to reproduce this result.
} 
proof of this result (for the sake of technical simplicity we add the assumption of diagonalizability of the matrix $A_{\infty}$ ) by presenting a reduction algorithm consisting of a number of elementary and explicitly written transformations.

It is a one-line calculation that shows that the $\mathcal{S}_{(n, m)}$ Schlesinger equations for the matrices $B_{1}(u), \ldots, B_{n}(u)$ of the form

$$
B_{i}(u)=\left(\begin{array}{cc}
B_{i}^{\prime}(u) & C_{i}(u) \\
0 & B_{i}^{\prime \prime}(u)
\end{array}\right),
$$

where $B_{i}^{\prime}(u)$ and $B_{i}^{\prime \prime}(u)$ are respectively $k \times k$ and $(m-k) \times(m-k)$ matrices, reduces to the $\mathcal{S}_{(n, k)}$ and $\mathcal{S}_{(n, m-k)}$ Schlesinger systems for the matrices $B_{i}^{\prime}(u)$ and $B_{i}^{\prime \prime}(u)$ and to the linear Pfaffian equations

$$
\begin{aligned}
\partial_{j} C_{i} & =\frac{1}{u_{i}-u_{j}}\left(B_{i}^{\prime} C_{j}-B_{j}^{\prime} C_{i}+C_{i} B_{j}^{\prime \prime}-C_{j} B_{i}^{\prime \prime}\right), \quad j \neq i \\
\partial_{i} C_{i} & =-\sum_{j \neq i} \frac{1}{u_{i}-u_{j}}\left(B_{i}^{\prime} C_{j}-B_{j}^{\prime} C_{i}+C_{i} B_{j}^{\prime \prime}-C_{j} B_{i}^{\prime \prime}\right) .
\end{aligned}
$$

Therefore the Schlesinger deformation of the reduced system (1.5) belongs to a Picard - Vessiot type extension of the composite $\mathcal{K}_{n, k} \mathcal{K}_{n, m-k}$.

To complete the proof of Theorem 1.1] we need to invert the above gauge transformation, i.e., to express the coefficients of the original Fuchsian system (1.4) via the solution of the reduced system (1.5).

Lemma 1.4. (i) For a Fuchsian system 1.4 satisfying the assumptions of the previous lemma, the monodromy data, in the sense of Definition [2.5 here below,

$$
\Lambda^{(1)}(A), R^{(1)}(A), \ldots, \Lambda^{(\infty)}(A), R^{(\infty)}(A), C_{1}(A), \ldots, C_{n}(A)
$$

of the system 1.4) and

$$
\Lambda^{(1)}(B), R^{(1)}(B), \ldots, \Lambda^{(\infty)}(B), R^{(\infty)}(B), C_{1}(B), \ldots, C_{n}(B)
$$

of (1.5) are related by

$$
\begin{aligned}
& \Lambda^{(i)}(B)=P^{-1} \Lambda^{(i)}(A) P, \quad i=1, \ldots, n \\
& R^{(i)}(B)=P^{-1} R^{(i)}(A) P, \quad i=1, \ldots, \infty \\
& C^{(i)}(B)=P^{-1} C^{(i)}(A) P, \quad i=1, \ldots, n \\
& \Lambda^{(\infty)}(B)=P^{-1} \Lambda^{(\infty)}(A) P+\operatorname{diag}\left(N_{1}, \ldots, N_{m}\right), \quad N_{i} \in \mathbb{Z} .
\end{aligned}
$$

Here $P \in S_{m}$ is a permutation matrix.

(ii) Denote $\mathcal{K}_{n, m}^{A}$ and $\mathcal{K}_{n, m}^{B}$ the differential fields associated with the Schlesinger deformations of two systems (1.4) and (1.5) respectively. If the monodromy data of the systems are related as in (1.6) then there exists a matrix

$$
\tilde{G}(z ; u) \in \overline{\mathcal{K}}_{n, m}^{B}[z], \quad \operatorname{det} \tilde{G}(z ; u) \equiv 1
$$


such that

$$
A(z) \equiv \tilde{G}^{-1}(z ; u) B(z ; u) \tilde{G}(z ; u)+\tilde{G}^{-1}(z ; u) \frac{d \tilde{G}(z ; u)}{d z} .
$$

Here, like in Lemma 1.3, $\overline{\mathcal{K}}_{n, m}^{B}$ is a suitable Picard - Vessiot type extension of the field $\mathcal{K}_{n, m}^{B}$.

We obtain therefore two inclusions

$$
\mathcal{K}_{n, m}^{B} \subset \overline{\mathcal{K}}_{n, m}^{A}, \quad \mathcal{K}_{n, m}^{A} \subset \overline{\mathcal{K}}_{n, m}^{B} .
$$

The Theorem 1.1 easily follows from the above statements.

Let us proceed now to the second mechanism of reducing the Schlesinger equations. Let us assume that $l$ monodromy matrices $M_{i_{1}}, \ldots, M_{i_{l}}$ of the Fuchsian system of the form (1.2), are scalar matrices (i.e., they are proportional to $\mathbb{1}$ ). In that case we will call the solution $A_{1}(u), \ldots A_{n}(u)$ is $l$-smaller. We call l-erased the Fuchsian system $\mathcal{S}_{n-l, m}$ of the same size with the poles $z=u_{i_{1}}, \ldots, z=u_{i_{l}}$ erased.

Theorem 1.5. Let $A_{1}, \ldots, A_{n}$ be a l-smaller solution of the Schlesinger equations. Then $A_{1}(u), \ldots, A_{n}(u)$ belong to the differential field obtained by admissible extensions from $\mathcal{K}_{(n-l, m)}$, the rational closure of the differential field $\mathcal{S}_{n-l, m}$ associated with a solution to the l-erased Fuchsian system $S_{(n-l, m)}$. In particular, if $l=n-2$ then $A_{1}, \ldots, A_{n}$ belong to the differential field obtained by admissible extensions from $\mathbb{C}\left(u_{1}, \ldots, u_{n}\right)$.

The proof of this Theorem consists first in observing that, due to the fact that all matrices $A_{1}, \ldots, A_{n}$ can be assumed to be traceless, any scalar matrix $M_{k}$ must have the form

$$
M_{k}=e^{\frac{2 \pi i p}{m}} \mathbb{1}, \quad p \in \mathbb{Z} .
$$

As a first step we assume $M_{k}$, say for $k=n$, to be the identity and we construct a gauge transformation in a suitable Picard - Vessiot type extension of the field $\mathcal{K}_{n, m}^{A}$ defined by the $l$-solution $A_{1}, \ldots, A_{n}$, which maps $A_{n}$ to zero without changing the nature of the other singular points $u_{1}, \ldots, u_{n-1}$, nor introducing new ones. In this way we obtain a new solution $B_{1}, \ldots, B_{n-1}$ of the Schlesinger equations $S_{n-1, m}$. We then prove that the original solution $A_{1}, \ldots, A_{n}$ can be constructed in terms of $B_{1}, \ldots, B_{n-1}$ by means of admissible operations.

When $M_{k}=e^{\frac{2 \pi i p}{m}} \mathbb{1}$ is not the identity, we need to map $A_{1}, \ldots, A_{n}$ birationally to a new solution $\tilde{A}_{1}, \ldots, \tilde{A}_{n}$ of the Schlesinger equations with $\tilde{M}_{k}=$ $\mathbb{1}$. To this end we apply the birational canonical transformations of Schlesinger equations found in $[8]^{3}$.

\footnotetext{
${ }^{3}$ An alternative way, as it was proposed by the referee, would be to replace our canonical transformations by a combination of Schlesinger transformations of [18 with scalar shifts
} 
To present here this class of transformations let us briefly remind the canonical Hamiltonian formulation of Schlesinger equations $S_{(n, m)}$ of $[8]$.

Recall [19, 25] that Schlesinger equations can be written as Hamiltonian systems on the Lie algebra

$$
\mathfrak{g}:=\oplus_{i=1}^{n} g l(m) \ni\left(A_{1}, \ldots, A_{n}\right)
$$

with respect to the standard linear Lie - Poisson bracket on $\mathfrak{g}$ with some quadratic time-dependent Hamiltonians of the form

$$
H_{k}:=\sum_{l \neq k} \frac{\operatorname{tr}\left(A_{k} A_{l}\right)}{u_{k}-u_{l}} .
$$

Because of isomonodromicity they can be restricted onto the symplectic leaves

$$
\mathcal{O}_{1} \times \cdots \times \mathcal{O}_{n} \in \mathfrak{g}
$$

obtained by fixation of the conjugacy classes $\mathcal{O}_{1}, \ldots, \mathcal{O}_{n}$ of the matrices $A_{1}$, $\ldots, A_{n}$. The matrix $A_{\infty}$ given in (1.3) is a common integral of the Schlesinger equations. Applying the procedure of symplectic reduction [26] we obtain the reduced symplectic space

$$
\left\{A_{1} \in \mathcal{O}_{1}, \ldots, A_{n} \in \mathcal{O}_{n}, A_{\infty}=\text { given diagonal matrix }\right\}
$$
modulo simultaneous diagonal conjugations.

The dimension of this reduced symplectic leaf in the generic situation is equal to $2 g$ where

$$
g=\frac{m(m-1)(n-1)}{2}-(m-1) .
$$

In 8 a new system of the so-called isomonodromic Darboux coordinates $q_{1}$, $\ldots, q_{g}, p_{1}, \ldots, p_{g}$ on generic symplectic manifolds (1.9) was constructed and the new Hamiltonians were expressed in these coordinates. Let us explain this construction.

The Fuchsian system (1.2) can be reduced to a scalar differential equation of the form

$$
y^{(m)}=\sum_{l=0}^{m-1} d_{l}(z) y^{(l)}
$$

For example, one can eliminate last $m-1$ components of the vector function $\Phi$ to obtain a $m$-th order equation for the first component $y:=\Phi_{1}$. (Observe that the reduction procedure depends on the choice of the component of $\Phi$.) The resulting Fuchsian equation will have regular singularities at the same points $z=u_{1}, \ldots, z=u_{n}, z=\infty$. It will also have other singularities produced by the reduction procedure. However, they will be apparent singularities, i.e., the solutions to (1.10) will be analytic in these points. Generically there will be exactly $g$ apparent singularities (cf. [31; a more precise result about the

instead. However, the birationality of the proposed transformation need to be justified in the resonant case. 
number of apparent singularities working also in the nongeneric situation was obtained in [3]); they are the first part $q_{1}, \ldots, q_{g}$ of the canonical coordinates. The conjugated momenta are defined by

$$
p_{i}=\operatorname{Res}_{z=q_{i}}\left(d_{m-2}(z)+\frac{1}{2} d_{m-1}^{2}(z)\right), \quad i=1, \ldots, g .
$$

Theorem 1.6. [8] Let the eigenvalues of the matrices $A_{1}, \ldots, A_{n}, A_{\infty}$ be pairwise distinct. Then the map

$$
\left\{\begin{array}{c}
\text { Fuchsian systems with given poles, } \\
\text { given eigenvalues of } A_{1}, \ldots, A_{n}, A_{\infty} \\
\text { modulo diagonal conjugations }
\end{array}\right\} \rightarrow\left(q_{1}, \ldots, q_{g}, p_{1}, \ldots, p_{g}\right)
$$

gives a system of rational Darboux coordinates on the generic reduced symplectic leaf (1.9). The Schlesinger equations $S_{(n, m)}$ in these coordinates are written in the canonical Hamiltonian form

$$
\begin{aligned}
\frac{\partial q_{i}}{\partial u_{k}} & =\frac{\partial \mathcal{H}_{k}}{\partial p_{i}} \\
\frac{\partial p_{i}}{\partial u_{k}} & =-\frac{\partial \mathcal{H}_{k}}{\partial q_{i}}
\end{aligned}
$$

with the Hamiltonians

$$
\mathcal{H}_{k}=\mathcal{H}_{k}(q, p ; u)=-\operatorname{Res}_{z=u_{k}}\left(d_{m-2}(z)+\frac{1}{2} d_{m-1}^{2}(z)\right), \quad k=1, \ldots, n .
$$

Here rational Darboux coordinates means that the elementary symmetric functions $\sigma_{1}(q), \ldots, \sigma_{g}(q)$ and $\sigma_{1}(p), \ldots, \sigma_{g}(p)$ are rational functions of the coefficients of the system and of the poles $u_{1}, \ldots, u_{n}$. Moreover, there exists a section of the map (1.11) given by rational functions

$$
A_{i}=A_{i}(q, p), \quad i=1, \ldots, n,
$$

symmetric in $\left(q_{1}, p_{1}\right), \ldots,\left(q_{g}, p_{g}\right)$ with coefficients depending on $u_{1}, \ldots, u_{n}$ and on the eigenvalues if the matrices $A_{i}, i=1, \ldots, n, \infty$. All other Fuchsian systems with the same poles $u_{1}, \ldots, u_{n}$, the same eigenvalues and the same $\left(p_{1}, \ldots, p_{g}, q_{1}, \ldots, q_{g}\right)$ are obtained by simultaneous diagonal conjugation

$$
A_{i}(q, p) \mapsto C^{-1} A_{i}(q, p) C, \quad i=1, \ldots, n, \quad C=\operatorname{diag}\left(c_{1}, \ldots, c_{m}\right) .
$$

Theorem 1.7. 8] The Schlesinger equations $S_{(n, m)}$ written in the canonical form of Theorem 1.3 admit a group of birational canonical transformations $\left\langle S_{2}, \ldots, S_{m}, S_{\infty}\right\rangle$

$$
S_{k}:\left\{\begin{array}{l}
\tilde{q}_{i}=u_{1}+u_{k}-q_{i}, \quad i=1, \ldots, g \\
\tilde{p}_{i}=-p_{i}, \quad i=1, \ldots, g \\
\tilde{u}_{l}=u_{1}+u_{k}-u_{l}, \quad l=1, \ldots, n \\
\tilde{\mathcal{H}}_{l}=-\mathcal{H}_{l}, \quad l=1, \ldots, n
\end{array}\right.
$$




$$
S_{\infty}:\left\{\begin{array}{l}
\tilde{q}_{i}=\frac{1}{q_{i}-u_{1}}, \quad i=1, \ldots, g, \\
\tilde{p}_{i}=-p_{i} q_{i}^{2}-\frac{2 m^{2}-1}{m} q_{i}, \quad i=1, \ldots, g, \\
\tilde{u}_{l}=\frac{1}{u_{l}-u_{1}}, \quad l=2, \ldots, n, \\
u_{1} \mapsto \infty, \\
\infty \mapsto u_{1}, \\
\tilde{H}_{1}=H_{1}, \\
\tilde{H}_{l}=-H_{l}\left(u_{l}-u_{1}\right)^{2}+\left(u_{l}-u_{1}\right)\left(d_{m-1}^{0}\left(u_{l}-u_{1}\right)\right)^{2}- \\
\quad-\left(u_{l}-u_{1}\right) \frac{(m-1)\left(m^{2}-m-1\right)}{m} d_{m-1}^{0}\left(u_{l}-u_{1}\right), \\
\text { for } \quad l=2, \ldots, n
\end{array}\right.
$$

where

$$
d_{m-1}^{0}\left(u_{k}\right)=\sum_{s=1}^{g} \frac{1}{u_{k}-q_{s}}-\frac{m(m-1)}{2} \sum_{l \neq k} \frac{1}{u_{k}-u_{l}} .
$$

The transformation $S_{k}$ acts on the monodromy matrices as follows

$$
\begin{aligned}
& \tilde{M}_{1}=M_{1}^{-1} \ldots . . M_{k-1}^{-1} M_{k} M_{k-1} \ldots M_{1}, \\
& \tilde{M}_{j}=M_{j-1}, \quad j=2, \ldots, k, \\
& \tilde{M}_{i}=M_{i}, \quad i=k+1, \ldots, n .
\end{aligned}
$$

The transformation $S_{\infty}$ acts on the monodromy matrices as follows

$$
\begin{aligned}
& \tilde{M}_{\infty}=e^{-\frac{2 \pi i}{m}} M_{1}, \quad \tilde{M}_{1}=e^{\frac{2 \pi i}{m}} M_{\infty}, \\
& \tilde{M}_{j}=M_{1}^{-1} M_{j} M_{1} \quad \text { for } \quad j=2, \ldots, n .
\end{aligned}
$$

To conclude, Theorems 1.1 and 1.2 show that for certain very special monodromy groups the Schlesinger equations $S_{(n, m)}$ reduce to solutions of $S_{\left(n^{\prime}, m^{\prime}\right)}$ with $n^{\prime}<n$ and/or $m^{\prime}<m$. We do not know any other general mechanism of reducibility of Schlesinger equations. As generically the monodromy group of the system (1.2) is not reducible nor smaller, we expect that generic solutions of the Schlesinger equations $S_{(n, m)}$ do not belong to any admissible extension of composites of the differential fields of the form $\mathcal{K}_{\left(n^{\prime}, m^{\prime}\right)}$ with $n^{\prime}<n$ and/or $m^{\prime}<m$. The proof of this fact, that is the proof of irreducibility of the Schlesinger equations, is still a rather intriguing open problem.

\section{SChlesinger EQUATIONS AS MONODROMY PRESERVING DEFORMATions OF FUCHSIAN SYSTEMS.}

In this section we establish our notations, remind a few basic definitions and prove some technical lemmata that will be useful throughout this paper.

The Schlesinger equations $\mathcal{S}_{(n, m)}$ describe monodromy preserving deformations of Fuchsian systems (1.2) with $n+1$ regular singularities at $u_{1}, \ldots, u_{n}$, 
$u_{n+1}=\infty:$

$$
\frac{\mathrm{d}}{\mathrm{d} z} \Phi=\sum_{k=1}^{n} \frac{A_{k}}{z-u_{k}} \Phi, \quad z \in \mathbb{C} \backslash\left\{u_{1}, \ldots, u_{n}\right\}
$$

$A_{k}$ being $m \times m$ matrices independent of $z$, and $u_{k} \neq u_{l}$ for $k \neq l, k, l=$ $1, \ldots, n+1$. Let us explain the precise meaning of this claim.

\subsection{Levelt basis near a logarithmic singularity and local monodromy} data. A system

$$
\frac{d \Phi}{d z}=\frac{A(z)}{z-z_{0}} \Phi
$$

is said to have a logarithmic, or Fuchsian singularity at $z=z_{0}$ if the $m \times m$ matrix valued function $A(z)$ is analytic in some neighborhood of $z=z_{0}$. By definition the local monodromy data of the system is the class of equivalence of such systems w.r.t. local gauge transformations

$$
A(z) \mapsto G^{-1}(z) A(z) G(z)+\left(z-z_{0}\right) G^{-1}(z) \partial_{z} G(z)
$$

analytic near $z=z_{0}$ satisfying

$$
\operatorname{det} G\left(z_{0}\right) \neq 0 \text {. }
$$

The local monodromy can be obtained by choosing a suitable fundamental matrix solution of the system (2.2). The most general construction of such a fundamental matrix was given by Levelt [21]. We will briefly recall this construction in the form suggested in 6 .

Without loss of generality one can assume that $z_{0}=0$. Expanding the system near $z=0$ one obtains

$$
\frac{d \Phi}{d z}=\left(\frac{A_{0}}{z}+A_{1}+z A_{2}+\ldots\right) \Phi .
$$

Let us now describe the structure of local monodromy data.

Two linear operators $\Lambda, R$ acting in the complex $m$-dimensional space $V$

$$
\Lambda, R: V \rightarrow V
$$

are said to form an admissible pair if the following conditions are fulfilled.

1. The operator $\Lambda$ is semisimple and the operator $R$ is nilpotent.

2. $R$ commutes with $e^{2 \pi i \Lambda}$,

$$
e^{2 \pi i \Lambda} R=R e^{2 \pi i \Lambda} .
$$

Observe that, due to the last condition the operator $R$ satisfies

$$
R\left(V_{\lambda}\right) \subset \oplus_{k \in \mathbb{Z}} V_{\lambda+k} \quad \text { for } \quad \text { any } \quad \lambda \in \operatorname{Spec} \Lambda,
$$

where $V_{\lambda} \subset V$ is the subspace of all eigenvectors of $\Lambda$ with the eigenvalue $\lambda$. The last condition says that

3. The sum in the r.h.s. of (2.6) contains only non-negative values of $k$. 
A decomposition

$$
R=R_{0}+R_{1}+R_{2}+\ldots
$$

is defined where

$$
R_{k}\left(V_{\lambda}\right) \subset V_{\lambda+k} \quad \text { for } \quad \text { any } \quad \lambda \in \operatorname{Spec} \Lambda .
$$

Clearly this decomposition contains only finite number of terms. Observe the useful identity

$$
z^{\Lambda} R z^{-\Lambda}=R_{0}+z R_{1}+z^{2} R_{2}+\ldots
$$

Theorem 2.1. For a system (2.4) with a logarithmic singularity at $z=0$ there exists a fundamental matrix solution of the form

$$
\Phi(z)=\Psi(z) z^{\Lambda} z^{R}
$$

where $\Psi(z)$ is a matrix valued function analytic near $z=0$ satisfying

$$
\operatorname{det} \Psi(0) \neq 0
$$

and $\Lambda, R$ is an admissible pair.

The formula (2.10) makes sense after fixing a branch of logarithm $\log z$ near $z=0$. Note that $z^{R}$ is a polynomial in $\log z$ due to nilpotency of $R$.

The proof can be found in 21] (cf. 6]). Clearly $\Lambda$ is the semisimple part of the matrix $A_{0} ; R_{0}$ coincides with its nilpotent part. The remaining terms of the expansion appear only in the resonant case, i.e., if the difference between some eigenvalues of $\Lambda$ is a positive integer. In the important particular case of a diagonalizable matrix $A_{0}$,

$$
T^{-1} A_{0} T=\Lambda=\operatorname{diag}\left(\lambda_{1}, \ldots, \lambda_{m}\right)
$$

with some nondegenerate matrix $T$, the matrix function $\Psi(z)$ in the fundamental matrix solution (2.10) can be obtained in the form

$$
\Psi(z)=T\left(\mathbb{1}+z \Psi_{1}+z^{2} \Psi_{2}+\ldots\right) .
$$

The matrix coefficients $\Psi_{1}, \Psi_{2}, \ldots$ of the expansion as well as the components $R_{1}, R_{2}, \ldots$ of the matrix $R$ (see (2.7) can be found recursively from the equations

$$
\left[\Lambda, \Psi_{k}\right]-k \Psi_{k}=-B_{k}+R_{k}+\sum_{i=1}^{k-1} \Psi_{k-i} R_{i}-B_{i} \Psi_{k-i}, \quad k \geq 1 .
$$

Here

$$
B_{k}:=T^{-1} A_{k} T, \quad k \geq 1 .
$$

If $k_{\max }$ is the maximal integer among the differences $\lambda_{i}-\lambda_{j}$ then

$$
R_{k}=0 \text { for } k>k_{\max } \text {. }
$$

Observe that vanishing of the logarithmic terms in the fundamental matrix solution (2.10) is a constraint imposed only on the first $k_{\max }$ coefficients $A_{1}$, $\ldots, A_{k_{\max }}$ of the expansion (2.4). 
Example 2.2. For the Fuchsian system (1.2) having diagonal the matrix

$$
A_{\infty}=-\left(A_{1}+\cdots+A_{n}\right)=\operatorname{diag}\left(\lambda_{1}, \ldots, \lambda_{m}\right)
$$

the fundamental matrix of Theorem 2.1 has the form

$$
\Phi=\left(1+\frac{\Psi_{1}}{z}+O\left(\frac{1}{z^{2}}\right)\right) z^{-\Lambda} z^{-R},
$$

where

$$
\begin{gathered}
\Lambda=A_{\infty}, \quad R=R_{1}+R_{2}+\ldots, \\
\left(R_{1}\right)_{i j}=\left\{\begin{array}{cc}
\left(B_{1}\right)_{i j}, & \lambda_{i}=\lambda_{j}+1 \\
0, & \text { otherwise }
\end{array}\right. \\
B_{1}=-\sum_{k} A_{k} u_{k} \\
\left(\Psi_{1}\right)_{i j}=\left\{\begin{array}{cc}
-\frac{\left(B_{1}\right)_{i j}}{\lambda_{i}-\lambda_{j}-1}, & \lambda_{i} \neq \lambda_{j}+1 \\
\operatorname{arbitrary}, & \text { otherwise }
\end{array}\right. \\
\left(R_{2}\right)_{i j}=\left\{\begin{array}{cc}
\left(B_{2}-\Psi_{1} R_{1}+B_{1} \Psi_{1}\right)_{i j}, & \lambda_{i}=\lambda_{j}+2 \\
0, & \text { otherwise }
\end{array}\right. \\
B_{2}=-\sum_{k} \begin{array}{cc}
A_{k} u_{k}^{2} \quad & \lambda_{i} \neq \lambda_{j}+2
\end{array} \\
\left(\Psi_{2}\right)_{i j}=\left\{\begin{array}{cc}
\frac{\left(-B_{2}+\Psi_{1} R_{1}-B_{1} \Psi_{1}\right)_{i j}}{\lambda_{i}-\lambda_{j}-2}, & \text { otherwise } \\
\text { arbitrary, }
\end{array}\right.
\end{gathered}
$$

etc.

It is not difficult to describe the ambiguity in the choice of the admissible pair of matrices $\Lambda, R$ describing the local monodromy data of the system (2.4). Namely, the diagonal matrix $\Lambda$ is defined up to permutations of diagonal entries. Assuming the order fixed, the ambiguity in the choice of $R$ can be described as follows [6]. Denote $\mathcal{C}_{0}(\Lambda) \subset G L(V)$ the subgroup consisting of invertible linear operators $G: V \rightarrow V$ satisfying

$$
z^{\Lambda} G z^{-\Lambda}=G_{0}+z G_{1}+z^{2} G_{2}+\ldots
$$

The definition of the subgroup can be reformulated [6] in terms of invariance of certain flag in $V$ naturally associated with the semisimple operator $\Lambda$. The matrix $\tilde{R}$ obtained from $R$ by the conjugation of the form

$$
\tilde{R}=G^{-1} R G
$$


will be called equivalent to $R$. Multiplying (2.10) on the right by $G$ one obtains another fundamental matrix solution to the same system of the same structure

$$
\tilde{\Phi}(z):=\Psi(z) z^{\Lambda} z^{R} G=\tilde{\Psi}(z) z^{\Lambda} z^{\tilde{R}}
$$

i.e., $\tilde{\Psi}(z)$ is analytic at $z=0$ with $\operatorname{det} \tilde{\Psi}(0) \neq 0$.

The columns of the fundamental matrix (2.10) form a distinguished basis in the space of solutions to (2.4).

Definition 2.3. The basis given by the columns of the matrix (2.10) is called Levelt basis in the space of solutions to (2.4). The fundamental matrix (2.10) is called Levelt fundamental matrix solution.

The monodromy transformation of the Levelt fundamental matrix solution reads

$$
\Phi\left(z e^{2 \pi i}\right)=\Phi(z) M, \quad M=e^{2 \pi i \Lambda} e^{2 \pi i R} .
$$

To conclude this Section let us denote $\mathcal{C}(\Lambda, R)$ the subgroup of invertible transformations of the form

$$
\mathcal{C}(\Lambda, R)=\left\{G \in G L(V) \mid z^{\Lambda} G z^{-\Lambda}=\sum_{k \in \mathbb{Z}} G_{k} z^{k} \text { and }[G, R]=0\right\} .
$$

The subgroups $\mathcal{C}(\Lambda, R)$ and $\mathcal{C}(\Lambda, \tilde{R})$ associated with equivalent matrices $R$ and $\tilde{R}$ are conjugated. It is easy to see that this subgroup coincides with the centralizer of the monodromy matrix (2.14)

$$
G \in \mathcal{C}(\Lambda, R) \quad \text { iff } \quad G e^{2 \pi i \Lambda} e^{2 \pi i R}=e^{2 \pi i \Lambda} e^{2 \pi i R} G, \quad \operatorname{det} G \neq 0 .
$$

Denote

$$
\mathcal{C}_{0}(\Lambda, R) \subset \mathcal{C}(\Lambda, R)
$$

the subgroup consisting of matrices $G$ such that the expansion (2.15) contains only non-negative powers of $z$. Multiplying the Levelt fundamental matrix (2.10) by a matrix $G \in \mathcal{C}_{0}(\Lambda, R)$ one obtains another Levelt solution to (2.4)

$$
\Psi(z) z^{\Lambda} z^{R} G=\tilde{\Psi}(z) z^{\Lambda} z^{R} .
$$

In the next Section we will see that the quotient $\mathcal{C}(\Lambda, R) / \mathcal{C}_{0}(\Lambda, R)$ plays an important role in the theory of monodromy preserving deformations.

2.2. Monodromy data and isomonodromic deformations of a Fuchsian system. Denote $\lambda_{j}^{(k)}, j=1, \ldots, m$, the eigenvalues of the matrix $A_{k}$, $k=1, \ldots, n, \infty$ where the matrix $A_{\infty}$ is defined as

$$
A_{\infty}:=-\sum_{k=1}^{n} A_{k} .
$$

For the sake of technical simplicity let us assume that

$$
\lambda_{i}^{(k)} \neq \lambda_{j}^{(k)} \text { for } \quad i \neq j, \quad k=1, \ldots, n, \infty .
$$


Moreover, it will be assumed that $A_{\infty}$ is a constant diagonal $m \times m$ matrix with eigenvalues $\lambda_{j}^{(\infty)}, j=1, \ldots, m$.

Denote $\Lambda^{(k)}, R^{(k)}$ the local monodromy data of the Fuchsian system near the points $z=u_{k}, k=1, \ldots, n, \infty$. The matrices $\Lambda^{(k)}$ are all diagonal

$$
\Lambda^{(k)}=\operatorname{diag}\left(\lambda_{1}^{(k)}, \ldots, \lambda_{m}^{(k)}\right), \quad k=1, \ldots, n, \infty .
$$

and, under our assumptions

$$
\Lambda^{(\infty)}=A_{\infty} .
$$

Recall that the matrix $G \in G L(m, \mathbb{C})$ belongs to the group $\mathcal{C}_{0}\left(\Lambda^{(\infty)}\right)$ iff

$$
z^{-\Lambda^{(\infty)}} G z^{\Lambda^{(\infty)}}=G_{0}+\frac{G_{1}}{z}+\frac{G_{2}}{z^{2}}+\ldots
$$

It is easy to see that our assumptions about the eigenvalues of $A_{\infty}$ imply diagonality of the matrix $G_{0}$.

Let us also remind that the matrices $\Lambda^{(k)}$ satisfy

$$
\operatorname{Tr} \Lambda^{(1)}+\cdots+\operatorname{Tr} \Lambda^{(\infty)}=0 .
$$

Definition 2.4. The numbers $\lambda_{1}^{(k)}, \ldots, \lambda_{m}^{(k)}$ are called the exponents of the system (1.2) at the singular point $u_{k}$.

Let us fix a fundamental matrix solutions of the form (2.10) near all singular points $u_{1}, \ldots, u_{n}, \infty$. To this end we are to fix branch cuts on the complex plane and choose the branches of $\operatorname{logarithms} \log \left(z-u_{1}\right), \ldots, \log \left(z-u_{n}\right)$, $\log z^{-1}$. We will do it in the following way: perform parallel branch cuts $\pi_{k}$ between $\infty$ and each of the $u_{k}, k=1, \ldots, n$ along a given (generic) direction. After this we can fix Levelt fundamental matrices analytic on

$$
z \in \mathbb{C} \backslash \cup_{k=1}^{n} \pi_{k},
$$

$\Phi_{k}(z)=T_{k}\left(\mathbb{1}+\mathcal{O}\left(z-u_{k}\right)\right)\left(z-u_{k}\right)^{\Lambda^{(k)}}\left(z-u_{k}\right)^{R^{(k)}}, \quad z \rightarrow u_{k}, \quad k=1, \ldots, n$ and

$$
\Phi(z) \equiv \Phi_{\infty}(z)=\left(\mathbb{1}+\mathcal{O}\left(\frac{1}{z}\right)\right) z^{-A_{\infty}} z^{-R^{(\infty)}}, \quad \text { as } \quad z \rightarrow \infty,
$$

Define the connection matrices by

$$
\Phi_{\infty}(z)=\Phi_{k}(z) C_{k},
$$

where $\Phi_{\infty}(z)$ is to be analytically continued in a vicinity of the pole $u_{k}$ along the positive side of the branch cut $\pi_{k}$.

The monodromy matrices $M_{k}, k=1, \ldots, n, \infty$ are defined with respect to a basis $l_{1}, \ldots, l_{n}$ of loops in the fundamental group

$$
\pi_{1}\left(\mathbb{C} \backslash\left\{u_{1}, \ldots u_{n}\right\}, \infty\right) .
$$


Choose the basis in the following way. The loop $l_{k}$ arrives from infinity in a vicinity of $u_{k}$ along one side of the branch cut $\pi_{k}$ that will be called positive, then it encircles $u_{k}$ going in anti-clock-wise direction leaving all other poles outside and, finally it returns to infinity along the opposite side of the branch cut $\pi_{k}$ called negative.

Denote $l_{j}^{*} \Phi_{\infty}(z)$ the result of analytic continuation of the fundamental matrix $\Phi_{\infty}(z)$ along the loop $l_{j}$. The monodromy matrix $M_{j}$ is defined by

$$
l_{j}^{*} \Phi_{\infty}(z)=\Phi_{\infty}(z) M_{j}, j=1, \ldots, n .
$$

The monodromy matrices satisfy

$$
M_{\infty} M_{n} \cdots M_{1}=\mathbb{1}, \quad M_{\infty}=\exp \left(2 \pi i A_{\infty}\right) \exp \left(2 \pi i R^{(\infty)}\right)
$$

if the branch cuts $\pi_{1}, \ldots, \pi_{n}$ enter the infinite point according to the order of their labels, i.e., the positive side of $\pi_{k+1}$ looks at the negative side of $\pi_{k}$, $k=1, \ldots, n-1$.

Clearly one has

$$
M_{k}=C_{k}^{-1} \exp \left(2 \pi i \Lambda^{(k)}\right) \exp \left(2 \pi i R^{(k)}\right) C_{k}, \quad k=1, \ldots, n .
$$

The collection of the local monodromy data $\Lambda^{(k)}, R^{(k)}$ together with the central connection matrices $C_{k}$ will be used in order to uniquely fix the Fuchsian system with given poles. They will be defined up to an equivalence that we now describe. The eigenvalues of the diagonal matrices $\Lambda^{(k)}$ are defined up to permutations. Fixing the order of the eigenvalues, we define the class of equivalence of the nilpotent part $R^{(k)}$ and of the connection matrices $C_{k}$ by factoring out the transformations of the form

$$
\begin{aligned}
& R_{k} \mapsto G_{k}^{-1} R_{k} G_{k}, \quad C_{k} \mapsto G_{k}^{-1} C_{k} G_{\infty}, \quad k=1, \ldots, n, \\
& G_{k} \in \mathcal{C}_{0}\left(\Lambda^{(k)}\right), \quad G_{\infty} \in \mathcal{C}_{0}\left(\Lambda^{(\infty)}\right) .
\end{aligned}
$$

Observe that the monodromy matrices (2.29) will transform by a simultaneous conjugation

$$
M_{k} \mapsto G_{\infty}^{-1} M_{k} G_{\infty}, \quad k=1,2, \ldots, n, \infty .
$$

Definition 2.5. The class of equivalence (2.30) of the collection

$$
\Lambda^{(1)}, R^{(1)}, \ldots, \Lambda^{(\infty)}, R^{(\infty)}, C_{1}, \ldots, C_{n}
$$

is called monodromy data of the Fuchsian system with respect to a fixed ordering of the eigenvalues of the matrices $A_{1}, \ldots, A_{n}$ and a given choice of the branch cuts.

Lemma 2.6. Two Fuchsian systems of the form (1.2) with the same poles $u_{1}, \ldots, u_{n}, \infty$ and the same matrix $A_{\infty}$ coincide, modulo diagonal conjugations if and only if they have the same monodromy data with respect to the same system of branch cuts $\pi_{1}, \ldots, \pi_{n}$. 
Proof. Let

$\Phi_{\infty}^{(1)}(z)=\left(\mathbb{1}+O\left(\frac{1}{z}\right)\right) z^{-\Lambda^{(\infty)}} z^{-R^{(\infty)}}, \quad \Phi_{\infty}^{(2)}(z)=\left(\mathbb{1}+O\left(\frac{1}{z}\right)\right) z^{-\tilde{\Lambda}^{(\infty)}} z^{-\tilde{R}^{(\infty)}}$

be the fundamental matrices of the form (2.25) of the two Fuchsian systems. Using assumption about $A_{\infty}$ we derive that $\tilde{\Lambda}^{(\infty)}=\Lambda^{(\infty)}$. Multiplying $\Phi_{\infty}^{(2)}(z)$ if necessary on the right by a matrix $G \in \mathcal{C}_{0}\left(\Lambda^{(\infty)}\right)$, we can obtain another fundamental matrix of the second system with

$$
\tilde{R}^{(\infty)}=R^{(\infty)} .
$$

Consider the following matrix:

$$
Y(z):=\Phi_{\infty}^{(2)}(z)\left[\Phi_{\infty}^{(1)}(z)\right]^{-1} .
$$

$Y(z)$ is an analytic function around infinity:

$$
Y(z)=G_{0}+\mathcal{O}\left(\frac{1}{z}\right), \quad \text { as } z \rightarrow \infty
$$

where $G_{0}$ is a diagonal matrix. Since the monodromy matrices coincide, $Y(z)$ is a single valued function on the punctured Riemann sphere $\overline{\mathbb{C}} \backslash\left\{u_{1}, \ldots, u_{n}\right\}$. Let us prove that $Y(z)$ is analytic also at the points $u_{k}$. Indeed, having fixed the monodromy data, we can choose the fundamental matrices $\Phi_{k}^{(1)}(z)$ and $\Phi_{k}^{(2)}(z)$ of the form (2.24) with the same connection matrices $C_{k}$ and the same matrices $\Lambda^{(k)}, R^{(k)}$. Then near the point $u_{k}, Y(z)$ is analytic:

$$
Y(z)=T_{k}^{(2)}\left(\mathbb{1}+\mathcal{O}\left(z-u_{k}\right)\right)\left[T_{k}^{(1)}\left(\mathbb{1}+\mathcal{O}\left(z-u_{k}\right)\right)\right]^{-1} .
$$

This proves that $Y(z)$ is an analytic function on all $\overline{\mathbb{C}}$ and then, by the Liouville theorem $Y(z)=G_{0}$, which is constant. So the two Fuchsian systems coincide, after conjugation by the diagonal matrix $G_{0}$.

Remark 2.7. The connection matrices are determined, within their equivalence classes by the monodromy matrices if the quotients $\mathcal{C}\left(\Lambda^{(k)}, R^{(k)}\right) / \mathcal{C}_{0}\left(\Lambda^{(k)}, R^{(k)}\right)$ are trivial for all $k=1, \ldots, n$. In particular this is the case when all the characteristic exponents at the poles $u_{1}, \ldots, u_{n}$ are non-resonant.

From the above Lemma the following result readily follows.

Theorem 2.8. If the matrices $A_{k}\left(u_{1}, \ldots, u_{n}\right)$ satisfy Schlesinger equations (1.1) and the matrix

$$
A_{\infty}=-\left(A_{1}+\cdots+A_{n}\right)
$$

is diagonal then all the characteristic exponents do not depend on $u_{1}, \ldots$, $u_{n}$. The fundamental matrix $\Phi_{\infty}(z ; u)$ can be chosen in such a way that the nilpotent matrix $R^{(\infty)}$ and also all the monodromy matrices are constant in $u_{1}$, $\ldots, u_{n}$. The coefficients of expansion of the fundamental matrix in $1 / z$ belong to a Picard - Vessiot type extension of the field $\mathcal{K}_{(n, m)}$ associated with the 
solution to Schlesinger equations. Moreover, the Levelt fundamental matrices $\Phi_{k}(z ; u)$ can be chosen in such a way that all the nilpotent matrices $R^{(k)}$ and also all the connection matrices $\mathcal{C}_{k}$ are constant. Viceversa, if the deformation $A_{k}=A_{k}\left(u_{1}, \ldots, u_{n}\right)$ is such that the monodromy data do not depend on $u_{1}$, $\ldots, u_{n}$ then the matrices $A_{k}\left(u_{1}, \ldots, u_{n}\right), k=1, \ldots, n$ satisfy Schlesinger equations.

Recall that the $u$-dependence of the needed fundamental matrix $\Phi_{\infty}(z ; u)$ is to be determined from the linear equations

$$
\partial_{i} \Phi_{\infty}(z ; u)=-\frac{A_{i}}{z-u_{i}} \Phi_{\infty}(z ; u), \quad i=1, \ldots, n,
$$

SO

$$
\begin{aligned}
& \partial_{i} \Psi_{1}=-A_{i} \\
& \partial_{i} \Psi_{2}=-A_{i} \Psi_{1}-u_{i} A_{i}
\end{aligned}
$$

etc.

Example 2.9. The following example shows that in general the coefficients of expansion of the fundamental matrix may not be in the field $\mathcal{K}_{(n, m)}$. Indeed, let us consider the following isomonodromic deformation of the Fuchsian system

$$
\begin{gathered}
\frac{d \Phi}{d z}=\left[\frac{A_{1}}{z}+\frac{A_{2}}{z-x}+\frac{A_{3}}{z-1}\right] \Phi, \\
A_{1}=\left(\begin{array}{l}
-\frac{(\sqrt{x}+1)^{2}}{16 \sqrt{x}}-\frac{1}{2 \sqrt{x}} \\
\frac{(\sqrt{x}+1)^{4}}{128 \sqrt{x}} \\
\frac{(\sqrt{x}+1)^{2}}{16 \sqrt{x}}
\end{array}\right) \\
A_{2}=\left(\begin{array}{ll}
-\frac{3 \sqrt{x}-1}{16 \sqrt{x}} & \frac{1}{2(\sqrt{x}+1) \sqrt{x}} \\
-\frac{(\sqrt{x}+1)(3 \sqrt{x}-1)^{2}}{128 \sqrt{x}} & \frac{3 \sqrt{x}-1}{16 \sqrt{x}}
\end{array}\right) . \\
A_{3}=\left(\begin{array}{ll}
\frac{1}{16}(\sqrt{x}-3) & \frac{1}{2(\sqrt{x}+1)} \\
-\frac{1}{128}(\sqrt{x}-3)^{2}(\sqrt{x}+1) & \frac{1}{16}(3-\sqrt{x})
\end{array}\right) .
\end{gathered}
$$

In this case

$$
A_{\infty}=\left(\begin{array}{cc}
\frac{1}{2} & 0 \\
0 & -\frac{1}{2}
\end{array}\right), \quad R^{(\infty)}=R_{1}^{(\infty)}=\left(\begin{array}{cc}
0 & -\frac{1}{2} \\
0 & 0
\end{array}\right) .
$$

The fundamental matrix

$$
\Phi=\left(\mathbb{1}+\frac{\Psi_{1}}{z}+O\left(\frac{1}{z^{2}}\right)\right) z^{-A_{\infty}} z^{-R}
$$


satisfying also the equation

$$
\frac{\partial \Phi}{\partial x}=-\frac{A_{2}}{z-x} \Phi
$$

has

$$
\Psi_{1}=\left(\begin{array}{ll}
\frac{1}{16}(3 x-2 \sqrt{x}) & -\log (\sqrt{x}+1) \\
\frac{1}{128}\left(\frac{9 x^{2}}{2}+2 x^{3 / 2}-5 x+2 \sqrt{x}\right) & \frac{1}{16}(2 \sqrt{x}-3 x)
\end{array}\right) .
$$

This matrix does not belong to the field $\mathcal{K}_{3,2}$ isomorphic in this case to the field of rational functions in $\sqrt{x}$.

\section{Reductions of the Schlesinger systems.}

\subsection{Reducible monodromy groups.}

Definition 3.1. Given a Fuchsian system of the form (1.2), we say that its monodromy group $\left\langle M_{1}, \ldots, M_{n}\right\rangle$ is $l$-reducible, $0<l<m$ if the monodromy matrices admit a common invariant subspace $X_{l}$ of dimension $l$ in the space of solutions of the system (1.2).

In particular, if the monodromy group is l-reducible, then there exists a basis where all monodromy matrices have the form

$$
M_{k}=\left(\begin{array}{c|c}
\delta_{k} & \beta_{k} \\
\hline 0 & \gamma_{k}
\end{array}\right), \quad k=1, \ldots, n, \infty,
$$

where $\delta_{k}, \beta_{k}$ and $\gamma_{k}$ are respectively some $l \times l, l \times(m-l)$ and $(m-l) \times(m-l)$ matrices.

Given the above definition, we can proceed to the proof of Theorem 1.1

We begin with the proof of Lemma 1.3. Our proof, valid for the case of diagonalizable $A_{\infty}$, is based on the fact that the sum of the exponents of the invariant sub-space $X_{l}$ must always be a negative integer (see 2] Lemma 5.2.2). We will perform a sequence of gauge transformations which map such sum to zero. Let $\lambda_{1}^{(\infty)}, \ldots, \lambda_{m}^{(\infty)}$ be the eigenvalues of $A_{\infty}$ (which is assumed to be diagonal). By means of a permutation $P \in S_{m}$, we order the eigenvalues of $A_{\infty}$ as follows: the first $l$ eigenvalues correspond to the invariant sub-space $X_{l}$ and we order them in such a way that $\Re \lambda_{1} \geq \Re \lambda_{r}$, for all $r=2, \ldots, l$. Then we order the other eigenvalues in such a way that $\Re \lambda_{m} \leq \Re \lambda_{s}$ for all $s=l+1, \ldots, m-1$.

Let us fix a fundamental matrix $\Phi$ normalized at infinity

$$
\Phi_{\infty}=\left(\mathbb{1}+\frac{\Psi_{1}}{z}+\frac{\Psi_{2}}{z^{2}}+\mathcal{O}\left(\frac{1}{z^{3}}\right)\right) z^{-A_{\infty}} z^{-R^{(\infty)}},
$$

where $\Psi_{1}, \Psi_{2}$ and $R^{(\infty)}$ are given by formulae (2.11) in Example 2.2.

Consider the following gauge transformation $\Phi(z)=(I(z)+G) \tilde{\Phi}(z)$ where

$$
I(z):=\operatorname{Diagonal}(z, 0, \ldots, 0),
$$


and

$$
\begin{aligned}
& G_{m 1}=\Psi_{1_{m 1}}, \quad G_{1 m}=-\frac{1}{G_{m 1}}, \\
& \text { if } p \neq 1, m, \quad G_{p p}=1, \quad G_{1 p}=\Psi_{1_{m p}} G_{1 m}, \quad G_{p 1}=\Psi_{1_{p 1}}, \\
& \text { if } p, q \neq 1, p \neq q, \quad G_{p q}=0, \\
& G_{11}=G_{1 m} \Psi_{2_{m 1}}+\Psi_{1_{11}}, \quad \text { and } \quad G_{m m}=0 .
\end{aligned}
$$

Let us first observe that the entries of the matrix $G$ belong to an extension of the differential field $\mathcal{K}_{(n, m)}$ obtained by adding solutions of the linear equations (2.36), (2.37). In order to see that this gauge transformation always works let us show that $\Psi_{1_{m 1}}(u)$ is never identically equal to zero if at least one of the $(m, 1)$ matrix entries of the matrices $A_{1}(u), \ldots, A_{n}(u)$ is different from identical zero. Indeed, this follows from the equations (2.36).

Let us prove that this transformation maps the matrices $A_{1}, \ldots, A_{n}$ to new matrices $\tilde{A}_{1}, \ldots, \tilde{A}_{n}$ given by

$$
\tilde{A}_{k}:=\left(I\left(u_{k}\right)+G\right)^{-1} A_{k}\left(I\left(u_{k}\right)+G\right),
$$

such that

$$
\tilde{A}_{\infty}=-\sum_{k=1}^{n} \tilde{A}_{k}=\operatorname{diagonal}\left(\lambda_{1}^{(\infty)}+1, \lambda_{2}^{(\infty)}, \ldots, \lambda_{m-1}^{(\infty)}, \lambda_{m}^{(\infty)}-1\right)
$$

In fact $(I(z)+G)^{-1}=J(z)+G^{-1}$ where

$$
J(z):=\text { Diagonal }(0, \ldots, 0, z)
$$

therefore

$$
\tilde{A}_{k}:=G^{-1} A_{k} I\left(u_{k}\right)+G^{-1} A_{k} G+J\left(u_{k}\right) A_{k} I\left(u_{k}\right)+J\left(u_{k}\right) A_{k} G .
$$


Multiplying by $G$ from the left and summing on all $k$ we get that the condition (3.2) is satisfied if and only if

$$
\begin{aligned}
& \left(\begin{array}{ccl}
-g_{11} & \left(\lambda_{1}^{(\infty)}-\lambda_{2}^{(\infty)}\right) g_{12} & \ldots \\
\left(\lambda_{2}^{(\infty)}-\lambda_{1}^{(\infty)}-1\right) g_{21} & 0 & \cdots \\
\cdots & 0 & \cdots \\
\left(\lambda_{m}^{(\infty)}-\lambda_{1}^{(\infty)}-1\right) g_{m 1} & 0 & \cdots
\end{array}\right. \\
& \left.\begin{array}{ccc}
\ldots & \left(\lambda_{1}^{(\infty)}-\lambda_{m-1}^{(\infty)}\right) g_{1 m-1} & \left(\lambda_{1}^{(\infty)}-\lambda_{m}^{(\infty)}+1\right) g_{1 m} \\
\ldots & \ldots & 0 \\
\ldots & \ldots & 0 \\
\ldots & \ldots & 0
\end{array}\right)= \\
& =\left(\begin{array}{cccc}
\sum_{k} A_{k_{11}} u_{k} & 0 & \ldots & 0 \\
\ldots & 0 & \ldots & 0 \\
\sum_{k} A_{k_{m 1}} u_{k} & 0 & \ldots & 0
\end{array}\right)+\left(\begin{array}{cccc}
g_{1 m} \sum_{k} A_{k_{m 1}} u_{k}^{2} & 0 & \ldots & 0 \\
0 & 0 & \ldots & 0 \\
\ldots & 0 & \ldots & 0
\end{array}\right)+ \\
& +\left(\begin{array}{ccc}
g_{1 m} \sum_{s} \sum_{k} A_{k_{m s}} u_{k} g_{s 1} & \ldots & g_{1 m} \sum_{s} \sum_{k} A_{k_{m s}} u_{k} g_{s m} \\
0 & \ldots & 0 \\
\ldots & \ldots & \ldots \\
0 & \ldots & 0
\end{array}\right)
\end{aligned}
$$

Observe that in the non-resonant case, these formulae are clearly satisfied thanks to the fact that $\Psi_{1}, \Psi_{2}$ and $R^{(\infty)}$ are given by formulae (2.11) in Example 2.2 In the resonant case, we only need to prove that when there is a resonance of type $\lambda_{m}^{(\infty)}-\lambda_{p}^{(\infty)}=1$ or $\lambda_{p}^{(\infty)}-\lambda_{1}^{(\infty)}=1$ for any $p=$ $1, \ldots, m-1$, then the corresponding coefficients $\sum_{k} A_{k_{m p}} u_{k}$, and $\sum_{k} A_{k_{p 1}} u_{k}$ are zero. Observe that such entries coincide with the $(m, p)$ and $(p, 1)$ entries in the matrix $R_{1}^{(\infty)}$ defined in Section 2.1 (see the formulae (2.11) ). Due to our ordering of the eigenvalues, if $\lambda_{m}^{(\infty)}-\lambda_{p}^{(\infty)}=1$ then $p=1, \ldots, l$ and if $\lambda_{p}^{(\infty)}-\lambda_{1}^{(\infty)}=1$ then $p=l+1, \ldots, m$. This means that the corresponding $R_{1}^{(\infty)}$ must lie in the $l \times(m-l)$ lower left block, which is 0 by the hypothesis that the monodromy group is $l$-reducible.

Finally, if $\lambda_{m}^{(\infty)}-\lambda_{1}^{(\infty)}=2$, we find that the gauge transformation works only if

$$
\begin{gathered}
\left(\sum_{l=1}^{n} A_{l_{m 1}} u_{l}\right)\left(\sum_{l=1}^{n}\left(A_{l_{11}}-A_{l_{m m}}\right) u_{l}\right)-\sum_{l=1}^{n} A_{l_{m 1}} u_{l}^{2}- \\
-\sum_{p=2}^{m-1}\left(\sum_{l=1}^{n} A_{l_{m p}} u_{l}\right) G_{p 1}=0 .
\end{gathered}
$$

This is precisely the condition $\left(R_{2}^{(\infty)}\right)_{m 1}=0$, as it follows from (2.11). 
Let us prove that this gauge transformation preserves the Schlesinger equations. Differentiating $\tilde{A}_{k}$ w.r.t. $u_{j}$, with $j \neq k$ and using the Schlesinger equations for $A_{1}, \ldots, A_{n}$ we get:

$$
\begin{gathered}
\frac{\partial \tilde{A}_{k}}{\partial u_{j}}=\left[\tilde{A}_{k},\left(I\left(u_{k}\right)+G\right)^{-1} \frac{\partial G}{\partial u_{j}}+\frac{\left(I\left(u_{k}\right)+G\right)^{-1} A_{j}\left(I\left(u_{k}\right)+G\right)}{u_{k}-u_{j}}\right]= \\
=\frac{\left[\tilde{A}_{k}, \tilde{A}_{j}\right]}{u_{k}-u_{j}}+ \\
+\left[\tilde{A}_{k},\left(I\left(u_{k}\right)+G\right)^{-1}\left(\frac{\partial G}{\partial u_{j}}+\frac{A_{j}\left(I\left(u_{k}\right)-I\left(u_{j}\right)\right)-B_{k j} A_{j}\left(I\left(u_{j}\right)+G\right)}{u_{k}-u_{j}}\right)\right],
\end{gathered}
$$

where

$$
B_{k j}=\left(\begin{array}{cccc}
0 & \ldots & 0 & \frac{u_{k}-u_{j}}{g_{m 1}} \\
0 & \ldots & 0 & 0 \\
\ldots & \ldots & \ldots & \ldots \\
0 & \ldots & 0 & 0
\end{array}\right) .
$$

Given the formulae (3.1), it is straightforward to prove that the equation

$$
\frac{\partial G}{\partial u_{j}}+\frac{A_{j}\left(I\left(u_{k}\right)-I\left(u_{j}\right)\right)-B_{k j} A_{j}\left(I\left(u_{j}\right)+G\right)}{u_{k}-u_{j}}=0,
$$

is equivalent to the equations (2.36), (2.37). This proves that also $\tilde{A}_{1}, \ldots, \tilde{A}_{n}$ satisfy the Schlesinger equations.

Now let the sum of the exponents of the invariant sub-space $X_{l}$ be $-N$, where $N$ is a positive integer. By iterating the above gauge transformation $N$ times, we arrive at a new solution $\left(B_{1}, \ldots, B_{n}\right)$ of the Schlesinger equations $S_{(n, m)}$ such that the sum of the exponents of the invariant sub-space $X_{l}$ is zero and

$$
B_{\infty}=\text { Diagonal }\left(\lambda_{1}^{(\infty)}+N, \lambda_{2}^{(\infty)}, \ldots, \lambda_{m-1}^{(\infty)}, \lambda_{m}^{(\infty)}-N\right) .
$$

To conclude the proof of this lemma, let us prove that this new solution $\left(B_{1}, \ldots, B_{n}\right)$ is of the form

$$
B_{k_{i j}}=0, \quad \forall i=l+1, \ldots, n, \quad j=1, \ldots, l .
$$

In fact suppose by contradiction that $B_{k}$ are not in the above form. Then by Lemma 5.2.2. in [2], there exists a gauge transformation $P$, constant in $z$, such that the new residue matrices $\tilde{B}_{k}=P^{-1} B_{k} P$ have the form has the form

$$
\tilde{B}_{k_{i j}}=0, \quad \forall i=l+1, \ldots, n, \quad j=1, \ldots, l .
$$

In general $\tilde{B}_{\infty}$ won't be diagonal, but we can diagonalize it by a constant gauge transformation $Q$ preserving the block triangular form of $\tilde{B}_{1}, \ldots, \tilde{B}_{n}$. So we end up with

$$
\hat{B}_{\infty}=Q^{-1} P^{-1} B_{\infty} P Q, \quad \hat{B}_{k}=Q^{-1} P^{-1} B_{k} P Q,
$$


and since $Q^{-1} P^{-1} B_{\infty} P Q=B_{\infty}$, we have that $P Q$ is diagonal. But then if $B_{k}$ is not block triangular, $\hat{B}_{k}$ is not either, so we obtain a contradiction. Lemma 1.3 is proved.

Proof of Theorem 1.1 By Lemma 1.3 we obtained a gauge transformation mapping a solution $\left(A_{1}, \ldots, A_{n}\right)$ of the Schlesinger system $S_{n, m}$ with a $l$ reducible monodromy group to a solution $B_{1}, \ldots, B_{n}$ of the block triangular form. As it was explained in the Introduction, the solution $\left(B_{1}(u), \ldots, B_{n}(u)\right)$ belongs to a Picard-Vessiot type extension $\mathcal{K}^{(N)}$ for some $N$ of the composite

$$
\mathcal{K}=\mathcal{K}_{n, l} \mathcal{K}_{n, m-l} .
$$

So, to conclude the proof of this theorem, we need to prove Lemma 1.4

Let us prove the formulae (1.6). Our gauge transformation constructed in Lemma 1.3 is an iteration of elementary gauges transformation $\Phi=(I(z)+G) \tilde{\Phi}$ mapping the matrices $A_{1}, \ldots, A_{n}$ to new matrices $\tilde{A}_{1}, \ldots, \tilde{A}_{n}$ such that $\tilde{A}_{\infty}=$ $A_{\infty}+\operatorname{Diagonal}(1,0, \ldots, 0,-1)$.

Let us prove that each elementary gauge transformation preserves the normalization at infinity. More precisely, we prove that if we fix a fundamental matrix $\Phi$ normalized at infinity

$$
\Phi_{\infty}=\left(\mathbb{1}+\frac{\Psi_{1}}{z}+\frac{\Psi_{2}}{z^{2}}+\mathcal{O}\left(\frac{1}{z^{3}}\right)\right) z^{-A_{\infty}} z^{-R^{(\infty)}},
$$

then $\tilde{\Phi}=\left(J(z)+G^{-1}\right) \Phi_{\infty}=\left(\mathbb{1}+\mathcal{O}\left(\frac{1}{z}\right)\right) z^{-\tilde{A}_{\infty}} z^{-\tilde{R}^{(\infty)}}$, whith $\tilde{R}^{(\infty)}=R^{(\infty)}$.

In fact it is straightforward to prove that

$$
\begin{aligned}
&(J(z)+\left.G^{-1}\right)\left(\mathbb{1}+\frac{\Psi_{1}}{z}+\frac{\Psi_{2}}{z^{2}}+\mathcal{O}\left(\frac{1}{z^{3}}\right)\right) \operatorname{Diagonal}\left(z, 0, \ldots, 0, \frac{1}{z}\right)= \\
&=\chi_{1} z+\chi_{0}+\mathcal{O}\left(\frac{1}{z}\right)
\end{aligned}
$$

where all matrix elements of $\chi_{1}$ are zero apart from the $(m, 1)$ element which is

$$
\chi_{1_{m 1}}=\frac{1}{g_{1 m}}+\Psi_{1_{m 1}}
$$

and the matrix elements of $\chi_{0}$ are given by the following: for $p \neq 1, m$

and

$$
\begin{gathered}
\chi_{0_{p p}}=1, \quad \chi_{0_{p 1}}=-g_{p 1}+\Psi_{1_{p 1}}, \quad \chi_{0_{p m}}=-\frac{g_{1 p}}{g_{1 m}}+\Psi_{1_{m p}}, \\
\chi_{0_{11}}=\chi_{0_{m m}}=1,
\end{gathered}
$$

$$
\chi_{0_{m 1}}=-\frac{g_{11}-\sum_{p=2}^{m-1} g_{1 p} \Psi_{1_{p m}}-\Psi_{1_{11}}+\sum_{p=2}^{m-1} g_{1 p} \Psi_{1_{p 1}}}{g_{1 m}}+\Psi_{2_{1 m}} .
$$

Using the formulae (3.1) for $G$ it is easy to prove that all entries of $\chi_{0}$ and $\chi_{1}$ are zero. 
Therefore each elementary gauge transformation preserves the normalization at infinity and maps $A_{\infty}$ to

$$
\tilde{A}_{\infty}=A_{\infty}+\operatorname{Diagonal}(1,0, \ldots, 0,-1) .
$$

Since the fundamental matrix remains normalized at infinity and the gauge transformation $\Phi=(I(z)+G) \tilde{\Phi}$ is analytic over $\mathbb{C}$, all monodromy data $\Lambda^{(1)}(A), R^{(1)}(A), \ldots, \Lambda^{(n)}(A), R^{(n)}(A), C_{1}(A), \ldots, C_{n}(A)$ are preserved in each iteration. Finally we prove that $R^{(\infty)}=\tilde{R}^{(\infty)}$. Due to the above we only need to prove that if

$$
z^{-A_{\infty}} R^{(\infty)}(A) z^{A_{\infty}}=\frac{R_{1}}{z}+\frac{R_{2}}{z^{2}}+\ldots \ldots
$$

where $R_{1}, R_{2}, \ldots$ are some matrices defined in Section 2 , then the matrix

$$
z^{-B_{\infty}} R^{(\infty)}(A) z^{B_{\infty}}
$$

is also polynomial in $\frac{1}{z}$. Since $B_{\infty}=A_{\infty}+\operatorname{Diagonal}(N, 0, \ldots, 0,-N)$ we get

$$
\begin{aligned}
& z^{-B_{\infty}} R^{(\infty)}(A) z^{B}= \\
& \operatorname{Diagonal}\left(z^{-N}, 1, \ldots, 1, z^{N}\right) z^{-A_{\infty}} R^{(\infty)}(A) z^{A_{\infty}} \operatorname{Diagonal}\left(z^{N}, 1, \ldots, 1, z^{-N}\right)= \\
& \operatorname{Diagonal}\left(z^{-N}, 1, \ldots, 1, z^{N}\right)\left(\frac{R_{1}}{z}+\frac{R_{2}}{z^{2}}+\ldots \ldots\right) \operatorname{Diagonal}\left(z^{N}, 1, \ldots, 1, z^{-N}\right)= \\
& \operatorname{Pol}\left(\frac{1}{z}\right)+\operatorname{Div}(z),
\end{aligned}
$$

where $\operatorname{Pol}\left(\frac{1}{z}\right)$ and $\operatorname{Div}(z)$ are matrix values polynomials in $\frac{1}{z}$ and $z$ respectively. The matrix elements of the latter are of the form:

$$
\begin{gathered}
\operatorname{Div}_{p q}=0, \quad \text { for } \quad q \neq 1, p \neq m, \quad \operatorname{Div}_{11}=0, \quad \operatorname{Div}_{m m}=0, \\
\operatorname{Div}_{p 1}=\sum R_{k_{p 1}}^{(\infty)} z^{N-k}, \quad \text { for } \quad p \neq 1, m, \\
\operatorname{Div}_{m q}=\sum R_{k_{m q}}^{(\infty)} z^{N-k}, \quad \text { for } q \neq 1, m \\
\operatorname{Div}_{m 1}=\sum R_{k_{m 1}}^{(\infty)} z^{2 N-k} .
\end{gathered}
$$

Since the monodromy group is reducible, all the entries of $R_{k}^{(\infty)}$ involved in the above expressions are identically zero. Therefore $\operatorname{Div}(z) \equiv 0$ as we wanted to prove. This proves the relations (1.6).

Let us now prove the statement ii) of Lemma 1.4. Starting form the solution $\left(B_{1}, \ldots, B_{n}\right)$, we can reconstruct $\left(A_{1}, \ldots, A_{n}\right)$ by iterating another gauge transformation of the form $(J(z)+F)$ where $J(z)=\operatorname{Diagonal}(0, \ldots, 0, z)$ and

$$
\begin{aligned}
& F_{1 m}=\tilde{\Psi}_{1_{1 m}}, \quad F_{m 1}=-\frac{1}{\tilde{\Psi}_{1_{1 m}}}, \\
& \text { if } p \neq 1, m, \quad F_{p p}=1, \quad F_{m p}=\tilde{\Psi}_{1_{1 p}} G_{m 1}, \quad F_{p m}=\tilde{\Psi}_{1_{p m}}, \\
& \text { if } p, q \neq 1, p \neq q, \quad F_{p q}=0, \\
& F_{11}=0, \quad \text { and } \quad F_{m m}=F_{m 1} \Psi_{2_{1 m}}+\Psi_{1_{m m}} .
\end{aligned}
$$


This gauge transformation is always well defined because $\tilde{\Psi}_{1_{1 m}}$ is always nonzero (proof of this fact is analogous to the proof that $\Psi_{1_{m 1}}$ is never zero given above). Following the same computations as in the proof of Lemmata 1.3 and 1.4 it is easy to verify that this gauge transformation preserves the Schlesinger equations, the normalization of the fundamental matrix at infinity, $R^{(\infty)}$ and maps $\tilde{A}_{\infty}$ to

$$
A_{\infty}=\tilde{A}_{\infty}-\operatorname{Diagonal}(1,0, \ldots, 0,-1) .
$$

The above arguments complete the proof of Lemma 1.4 and, therefore of Theorem 1.1.

3.1.1. Upper triangular monodromy groups. In this section we deal with the case of upper triangular monodromy groups, that is there exists a basis where all monodromy matrices have the form

$$
M_{k_{i j}}=0, \quad \forall \quad i>j .
$$

To prove Corollary 1.2 we iterate the procedure of the proof of Lemma 1.3 at the first step we show that $\left(A_{1}, \ldots, A_{n}\right)$ is mapped by a rational gauge transformation to $\left(A_{1}^{(1)}, \ldots, A_{n}^{(1)}\right)$ of the form

$$
A_{k i 1}^{(1)}=0, \quad \forall i \neq 1, \quad \forall k=1, \ldots, n .
$$

At the $l$-th step we show that $\left(A_{1}, \ldots, A_{n}\right)$ is mapped by a rational gauge transformation to $\left(A_{1}^{(l)}, \ldots, A_{n}^{(l)}\right)$ of the form

$$
A_{k i j}^{(l)}=0, \quad i>j, j \leq l, \quad k=1, \ldots, n .
$$

At the $m$-th step we obtain that is mapped by a rational gauge transformation to

$$
\tilde{A}_{k_{i j}}:=A_{k_{i j}}^{(m)}=0, \quad \forall \quad i>j .
$$

Let us show that $\tilde{A}_{k_{i j}}\left(u_{1}, \ldots, u_{n}\right)$ belongs to the Picard-Vessiot type extension $\mathcal{K}^{(N)}$ for some $N$ of

$$
\mathcal{K}=\mathbb{C}\left(u_{1}, \ldots, u_{n}\right) .
$$

Clearly the diagonal elements $\tilde{A}_{k}$ are the eigenvalues $\lambda_{1}^{(k)}, \ldots \lambda_{m}^{(k)}$. The Schlesinger equations for $i \neq j$ read:

$$
\begin{aligned}
& \frac{\partial}{\partial u_{j}} \tilde{A}_{i_{p, p+q}} \frac{\lambda_{p+q}^{(j)}-\lambda_{p}^{(j)}}{u_{i}-u_{j}} \tilde{A}_{i_{p, p+q}}-\frac{\lambda_{p+q}^{(i)}-\lambda_{p}^{(i)}}{u_{i}-u_{j}} \tilde{A}_{j_{p, p+q}}+ \\
& +\sum_{s=1}^{q-1} \frac{\tilde{A}_{i_{p, p+s}} \tilde{A}_{j_{p+s, p+q}}-\tilde{A}_{j_{p, p+s}} \tilde{A}_{i_{p+s, p+q}}}{u_{i}-u_{j}}
\end{aligned}
$$


and for $i=j$

$$
\begin{aligned}
& \frac{\partial}{\partial u_{i}} \tilde{A}_{i_{p, p+q}}=-\sum_{j \neq i}\left[\frac{\lambda_{p+q}^{(j)}-\lambda_{p}^{(j)}}{u_{i}-u_{j}} \tilde{A}_{i_{p, p+q}}-\frac{\lambda_{p+q}^{(i)}-\lambda_{p}^{(i)}}{u_{i}-u_{j}} \tilde{A}_{j_{p, p+q}}+\right. \\
& \left.+\sum_{s=1}^{q-1} \frac{\tilde{A}_{i_{p, p+s}} \tilde{A}_{j_{p+s, p+q}}-\tilde{A}_{j_{p, p+s}} \tilde{A}_{i_{p+s, p+q}}}{u_{i}-u_{j}}\right]
\end{aligned}
$$

where for $q=1$ the sum $\sum_{s=1}^{q-1}$ is zero. It is clear that for each $q, 1 \leq q<$ $m-p$, the differential system for the matrix elements $A_{i_{p, p+q}}, i=1, \ldots, n$ is linear and it is Pfaffian integrable because the Schlesinger equations are Pfaffian integrable. In particular it is worth observing that for each $q, 1 \leq$ $q<m-p$, the homogeneous part of such differential system is the Lauricella hypergeometric system (see [16]).

3.2. An example. Consider the following solution $A_{1}, A_{2}, A_{3}$ of the Schlesinger equations in dimension $m=2$, where we have chosen $u_{1}=0, u_{2}=x, u_{3}=1$, with matrix entries:

$$
\begin{aligned}
& A_{1_{11}}= \frac{2 \log \frac{\sqrt{x}+1}{\sqrt{x}-1} \sqrt{x}\left(x^{2}+4 x-5\right)-4 x(4+3 x)-\left(\log \frac{\sqrt{x}+1}{\sqrt{x}-1}\right)^{2}(x-1)^{2}}{2\left((x-1) \log \frac{\sqrt{x}+1}{\sqrt{x}-1}-2 \sqrt{x}\right)^{2}}, \\
& A_{1_{12}}= \frac{\left(\log \frac{\sqrt{x}+1}{\sqrt{x}-1}\right)^{2}(x-1)^{2}+2 x(5+3 x)-\left(x^{2}+6 x-7\right) \sqrt{x} \log \frac{\sqrt{x}+1}{\sqrt{x}-1}}{4\left((x-1) \log \frac{\sqrt{x}+1}{\sqrt{x}-1}-2 \sqrt{x}\right)^{2}(x-1)} \times \\
& \times\left(6 \sqrt{x}(1+x)-\left(x^{2}+2 x-3\right) \log \frac{\sqrt{x}+1}{\sqrt{x}-1}\right) \\
& A_{2_{11}}= \frac{\left(\log \frac{\sqrt{x}+1}{\sqrt{x}-1}\right)^{2}(x-1)^{3}-4 x(7+x)-8 \log \frac{\sqrt{x}+1}{\sqrt{x}-1} \sqrt{x}\left(x^{2}-3 x+2\right)}{4\left((x-1) \log \frac{\sqrt{x}+1}{\sqrt{x}-1}-2 \sqrt{x}\right)^{2}(x-1)}, \\
& A_{1_{21}}=\frac{\left((x-1) \log \frac{\sqrt{x}+1}{\sqrt{x}-1}-2 \sqrt{x}\right)^{2}}{8\left((x-1) \log \frac{\sqrt{x}+1}{\sqrt{x}-1}-2 \sqrt{x}\right)^{2}(x-1)^{2}} \\
& \quad-\frac{\left(\log \frac{\sqrt{x}+1}{\sqrt{x}-1}\right)^{2}(x-1)^{3}-16 x-2\left(3 x^{2}-8 x+5\right) \sqrt{x} \log \frac{\sqrt{x}+1}{\sqrt{x}-1}}{\left.8 \sqrt{x}(3+x)+\left(x^{2}-4 x+3\right) \log \frac{\sqrt{x}+1}{\sqrt{x}-1}\right)} \times
\end{aligned}
$$




$$
\begin{gathered}
A_{2_{21}}=-\frac{4 \sqrt{x}}{\left((x-1) \log \frac{\sqrt{x}+1}{\sqrt{x}-1}-2 \sqrt{x}\right)^{2}} \\
A_{3_{11}}=\frac{1}{2}-A_{1_{11}}-A_{2_{11}}, \quad A_{3_{12}}=-A_{1_{12}}-A_{2_{12}}, \quad A_{3_{21}}=-A_{1_{21}}-A_{2_{21}}, \\
A_{1_{22}}=-A_{1_{11}}, \quad A_{2_{22}}=-A_{2_{11}}, \quad A_{3_{22}}=-A_{3_{11}} .
\end{gathered}
$$

This solution has a reducible monodromy group. Observe that

$$
A_{\infty}=\left(\begin{array}{cc}
-\frac{1}{2} & 0 \\
0 & \frac{1}{2}
\end{array}\right)
$$

is resonant. By applying our technique, it is straightforward to obtain a the new solution $B_{1}, B_{2}, B_{3}$ of the Schlesinger equations, gauge equivalent to $A_{1}, A_{2}, A_{3}$ in the upper triangular form:

$$
B_{1}=\left(\begin{array}{cc}
\frac{1}{2} & \frac{\sqrt{x}}{x-1} \\
0 & -\frac{1}{2}
\end{array}\right), \quad B_{2}=\left(\begin{array}{cc}
-\frac{1}{4} & \frac{\sqrt{x}}{(x-1)^{2}} \\
0 & \frac{1}{4}
\end{array}\right),
$$

and

$$
B_{3}=\left(\begin{array}{cc}
-\frac{3}{4} & -\frac{x \sqrt{x}}{(x-1)^{2}} \\
0 & \frac{3}{4}
\end{array}\right), \quad B_{\infty}=\left(\begin{array}{cc}
\frac{1}{2} & 0 \\
0 & -\frac{1}{2}
\end{array}\right) .
$$

This new solution is actually algebraic. This shows that the differential fields $\mathcal{K}_{3,2}^{A}$ and $\mathcal{K}_{3,2}^{B}$ associated with the solutions $A_{1}, A_{2}, A_{3}$ and $B_{1}, B_{2}, B_{3}$ respectively are not isomorphic.

3.3. Smaller monodromy groups. The proof of Theorem 1.5 is based on a few lemmata:

Lemma 3.2. Let $A_{1}, \ldots, A_{n}$ be a solution of the Schlesinger equations such that one of the monodromy matrices $\left(M_{1}, \ldots, M_{n}\right)$, say $M_{l}$, is proportional to the identity, then then there exists a solution $\tilde{A}_{1}, \ldots, \tilde{A}_{l-1}, \tilde{A}_{l+1}, \ldots \tilde{A}_{n}$ of the Schlesinger equations in $n-1$ variables with monodromy matrices $M_{1}, \ldots, M_{l-1}$, $M_{l+1}, \ldots, M_{n}$. The original solution $A_{1}, \ldots, A_{n}$ depends rationally on $\tilde{A}_{1}, \ldots$, $\tilde{A}_{l-1}, \tilde{A}_{l+1}, \ldots \tilde{A}_{n}, \tilde{\Phi}\left(u_{l}\right)$ and on $u_{l}$.

Proof. Let us first consider the case $M_{l}=\mathbb{1}$, for simplicity, $l=n$. This means that all eigenvalues $\lambda_{1}^{(n)}, \ldots \lambda_{m}^{(n)}$ of $A_{n}$ are integers and $R^{(n)}=0$. To eliminate the singularity $n$, we perform a confromal transformation $\zeta=\frac{1}{z-u_{n}}$. We obtain

$$
\frac{\mathrm{d} \Phi}{\mathrm{d} \zeta}=\left(\frac{A_{\infty}}{\zeta}+\sum_{k=1}^{n-1} \frac{A_{k}}{\zeta-\tilde{u}_{k}}\right) \Phi,
$$

where $\tilde{u}_{k}=\frac{1}{u_{k}-u_{n}}$, for $k \neq n$. The new residue matrix at infinity is $-A_{n}$. We perform a gauge transformation diagonalizing $A_{n}$ and use iterations of the 
gauge transformation of the form $(I(\zeta)+G)$ where $G$ is defined by formulae (3.3) to map all eigenvalues of $A_{n}$ to zero.

We have seen in the proof of Lemma 1.3 that this gauge transformation is always well defined and it works for $R^{(\infty)}=0$. Of course similar formulae can be given to map any $\lambda_{j}^{(\infty)}$ to $\lambda_{j}^{(\infty)}+1$ and any $\lambda_{i}^{(\infty)}$ to $\lambda_{i}^{(\infty)}-1$. After enough iterations we end up with a new Fuchsian system of the form

$$
\frac{\mathrm{d} \tilde{\Phi}}{\mathrm{d} \zeta}=\left(\frac{\tilde{A}_{\infty}}{\zeta}+\sum_{k=1}^{n-1} \frac{\tilde{A}_{k}}{\zeta-\tilde{u}_{k}}\right) \tilde{\Phi}
$$

such that the residue at infinity is $\tilde{A}_{n}=0$.

Now we perform the inverse conformal transformation, $z=\frac{1}{\zeta}+u_{n}$, we obtain

$$
\frac{\mathrm{d} \tilde{\Phi}}{\mathrm{d} z}=\sum_{k=1}^{n-1} \frac{\tilde{A}_{k}}{z-u_{k}} \tilde{\Phi}
$$

and the residue at infinity is $\tilde{A}_{\infty}$. We finally perform a gauge transformation diagonalizing $\tilde{A}_{\infty}$, so that the final Fuchsian system is

$$
\frac{\mathrm{d} \hat{\Phi}}{\mathrm{d} z}=\sum_{k=1}^{n-1} \frac{\hat{A}_{k}}{z-u_{k}} \hat{\Phi}
$$

where $\hat{A}_{\infty}=A_{\infty}$.

All the monodromy data of this new system coincide with the ones of the original system with matrices $A_{1}, \ldots, A_{n}, A_{\infty}$. The proof of this fact is very similar to the proof of statement ii of lemma 1.4 and we omit it.

The new matrices $\hat{A}_{1}, \ldots, \hat{A}_{n-1}$ satisfy the Schlesinger equations because the gauge transformations of the form $(I(z)+G)$ where $G$ is defined by the formulae (3.3) preserve the Schlesinger equations. Observe that since $\hat{A}_{n}$ is zero, $\hat{A}_{1}, \ldots, \hat{A}_{n-1}$ satisfy the Schlesinger equations $S_{n-1, m}$.

We now want to reconstruct the original solution $A_{1}, \ldots, A_{n}$ from $\hat{A}_{1}, \ldots, \hat{A}_{n-1}$.

Let us consider the Fuchsian system

$$
\frac{\mathrm{d} \hat{\Phi}}{\mathrm{d} z}=\sum_{k=1}^{n-1} \frac{\hat{A}_{k}}{z-u_{k}} \hat{\Phi} .
$$

Let us choose any point $u_{n} \neq u_{k}, k=1, \ldots, n-1$ and perform the constant gauge transformation $\hat{\Psi}=\hat{\Phi}\left(u_{n}\right)^{-1} \hat{\Phi}$, where $\hat{\Phi}\left(u_{n}\right)$ is the value at $z=u_{n}$ of

$$
\hat{\Phi}(z)\left(\mathbb{1}+\mathcal{O}\left(\frac{1}{z}\right)\right) z^{-A_{\infty}} z^{R^{(\infty)}} .
$$

Let us perform the conformal transformation $\zeta=\frac{1}{z-u_{n}}$,

$$
\hat{\Psi}(\zeta):=\hat{\Phi}\left(u_{n}\right)^{-1} \hat{\Phi}\left(\frac{1}{\zeta}+u_{n}\right) .
$$


Let us apply a product $F_{\infty}(\zeta)$ of gauge transformations of the form $(J(\zeta)+F)$, where $F$ is given by the formulae (3.4), to create a new non-zero residue matrix at infinity with integer entries $\lambda_{1}^{(n)}, \ldots \lambda_{m}^{(n)}$ :

$$
\hat{\Psi}(\zeta)=F_{\infty}(\zeta) \hat{\Psi}(\zeta)=F_{\infty}(\zeta) \hat{\Phi}\left(u_{n}\right)^{-1} \hat{\Phi}\left(\frac{1}{\zeta}+u_{n}\right)
$$

Let us now apply the conformal transformation $z=\frac{1}{\zeta}+u_{n}$ :

$$
\tilde{\Phi}(z):=F_{\infty}\left(\frac{1}{z-u_{n}}\right) \hat{\Phi}\left(u_{n}\right)^{-1} \hat{\Phi}(z) .
$$

We need now to diagonalize the new residue matrix at infinity

$$
\tilde{A}_{\infty}=F_{\infty}\left(u_{n}\right) \hat{\Phi}\left(u_{n}\right)^{-1} \hat{A}_{\infty} \hat{\Phi}\left(u_{n}\right) F_{\infty}\left(u_{n}\right)^{-1} .
$$

To do so we put

$$
\Phi(z):=\hat{\Phi}\left(u_{n}\right) F_{\infty}\left(u_{n}\right)^{-1} \hat{\Phi}(z) .
$$

The new residue matrices are

$B_{i}=\hat{\Phi}\left(u_{n}\right) F_{\infty}\left(u_{n}\right)^{-1} F_{\infty}\left(\frac{1}{u_{i}-u_{n}}\right) \hat{\Phi}\left(u_{n}\right)^{-1} \hat{A}_{i} \hat{\Phi}\left(u_{n}\right) F_{\infty}^{-1}\left(\frac{1}{u_{i}-u_{n}}\right) F_{\infty}\left(u_{n}\right) \hat{\Phi}\left(u_{n}\right)^{-1}$

for $i=1, \ldots, n-1$ and

$$
B_{n}=\hat{\Phi}\left(u_{n}\right) F_{\infty}^{-1}\left(u_{n}\right) \operatorname{diagonal}\left(\lambda_{1}^{(n)}, \ldots \lambda_{m}^{(n)}\right) F_{\infty}\left(u_{n}\right) \hat{\Phi}\left(u_{n}\right)^{-1} .
$$

The Fuchsian system with residue matrices $B_{1}, \ldots, B_{n}, B_{\infty}$ has the same exponents and the same monodromy data as the original system of residue matrices $A_{1}, \ldots, A_{n}, A_{\infty}$. Therefore, by the uniqueness lemma [2.6] $A_{1}, \ldots, A_{n}, A_{\infty}$ coincide with $B_{1}, \ldots, B_{n}, B_{\infty}$ up to diagonal conjugation.

As a consequence $A_{1}, \ldots, A_{n}$ depend rationally on $\hat{A}_{1}, \ldots, \hat{A}_{n-1}$, on $\hat{\Phi}\left(u_{n}\right)$ and $u_{n}$.

Now let us suppose that $M_{l}$ is only proportional to the identity. This means that all eigenvalues $\lambda_{1}^{(l)}, \ldots \lambda_{m}^{(l)}$ of $A_{l}$ are resonant. Since their sum is zero, the only possibility is $M_{l}=\exp \left(\frac{2 \pi i s}{m}\right) \mathbb{1}$ for some $s=1, \ldots, m-1$. To transform this matrix to the identity we use iterations of the symmetries (1.13), (1.14), to map our solution $A_{1}, \ldots, A_{n}$ to a solution $\hat{A}_{1}, \ldots, \hat{A}_{n}$ having $M_{n}=\mathbb{1}$. Since these symmetries are birational, $A_{1}, \ldots, A_{n}$ are rational functions of $\hat{A}_{1}, \ldots, \hat{A}_{n}$. Then we can apply the above procedure to kill $\hat{A}_{n}$.

Remark 3.3. Observe that in Lemma 3.2 for $M_{l}=\exp \left(\frac{2 \pi}{m}\right) \mathbb{1}$, the new solution $\tilde{A}_{1}, \ldots, \tilde{A}_{l-1}, \tilde{A}_{l+1}, \ldots \tilde{A}_{n}$ has monodromy matrices $M_{1}, \ldots, M_{l-1}, M_{l+1}$, $\ldots, M_{n}$, and a new monodromy matrix at infinity $\exp \left(-\frac{2 \pi}{m}\right) M_{\infty}$.

Lemma 3.4. Let $\left(A_{1}, \ldots A_{n}\right)$ be a solution of the Schlesinger equations with $M_{\infty}$ proportional to the identity $\mathbb{1}$, say $M_{\infty}=\exp \left(\frac{2 \pi i}{m}\right) \mathbb{1}$. Suppose that $M_{n}$ is not proportional to the identity, then there exists a solution $\tilde{A}_{1}, \ldots \tilde{A}_{n-1}$ of the Schlesinger equations with monodromy matrices

$$
\mathcal{C}_{n} M_{1} \mathcal{C}_{n}^{-1}, \ldots, \mathcal{C}_{n} M_{n-1} \mathcal{C}_{n}^{-1}
$$


and $\tilde{M}_{\infty}=\mathcal{C}_{n} \exp \left(-\frac{2 \pi i}{m}\right) M_{n} \mathcal{C}_{n}^{-1}, \mathcal{C}_{n}$ being the connection matrix of $M_{n}$. The given solution $A_{1}, \ldots A_{n}$ depends rationally on $\tilde{A}_{1}, \ldots, \tilde{A}_{n-1}, \tilde{\Phi}\left(u_{n}\right)$ and on $u_{n}$.

We perform a symmetry (1.14) (or a conformal transformation), in order to apply Lemma 3.2 to the case $M_{1}$ proportional to the identity.

End of the proof of Theorem [1.5. Suppose that $A_{1}, \ldots, A_{n}$ is a solution of the Schlesinger equations such that the collection of its monodromy matrices $M_{1}, \ldots, M_{n}, M_{\infty}$ is $l$-smaller. If none of the monodromy matrices being proportional to the identity is equal to $M_{\infty}$, we can simply conclude by $l$ iterations of Lemma 3.2 If $M_{\infty}$ is proportional to the identity, first we apply Lemma 3.4 then we iterate Lemma $3.2 l-1$ times. This concludes the proof of Theorem 1.5

\section{REFERENCES}

[1] F.V. Andreev and A.V. Kitaev. Transformations $R S_{4}^{2}(3)$ of the ranks $\leq 4$ and algebraic solutions of the sixth Painlevé equation. Comm. Math. Phys., 228:no. 1, 151-176, 2002.

[2] D.V. Anosov and A.A. Bolibruch. The Riemann-Hilbert Problem, volume E 22. Aspects of Mathematics, 1994.

[3] A.A. Bolibruch. The 21-st Hilbert problem for linear Fuchsian systems. Developments in mathematics: the Moscow school. Chapman and Hall, London, 1993.

[4] A.A. Bolibruch. On isomonodromic deformations of Fuchsian systems. J. Dynam. Control Systems, 3:no. 4, 589-604, 1997.

[5] O. Costin and R. D. Costin. Asymptotic properties of a family of solutions of the Painlevé equation VI. Int. Math. Res. Not., 2002:no.22:1167-1182, 2002.

[6] B. Dubrovin. Painlevé transcendents in Two-Dimensional Topological Field Theory. The Painlevé property One Century Later, pages 287-412, 1999.

[7] B. Dubrovin and M. Mazzocco. Monodromy of certain Painlevé-VI transcendents and reflection groups. Invent. Math., 141:55-147, 2000.

[8] B. Dubrovin and M. Mazzocco. Canonical structures of the Schlesinger systems. preprint math.DG/0311261, 2003.

[9] R. Fuchs. Ueber lineare homogene Differentialgleichungen zweiter Ordnung mit drei im Endlichen gelegenen wesentlich singulären Stellen. Math. Ann., 63:301-321, 1907.

[10] R. Garnier. Sur des équations différentielles du troisième ordre dont l'intégrale générale est uniforme et sur une classe d'équations nouvelles d'ordre supérieur dont l'intégrale générale a ses points critiques fixes. Ann. Sci. École Norm. Sup., 29:no. 3, 1-126, 1912.

[11] R. Garnier. Solution du probleme de Riemann pour les systemes différentielles linéaires du second ordre. Ann. Sci. École Norm.. Sup., 43:239-352, 1926.

[12] D. Guzzetti. The elliptic representation of the general Painlevé VI equation. Comm. Pure Appl. Math., 55:no. 10, 1280-1363, 2002.

[13] N. Hitchin. A lecture on the octahedron. Bull. London Math. Soc., 35:no.5, 577-600, 2003.

[14] E.L. Ince. Ordinary differential equations. Dover Publications INC., 1956.

[15] A.R. Its and V.Yu. Novokshenov. The isomonodromic deformation method in the theory of Painlevé equations, volume 1191 of Lecture notes in mathematics. Springer, 1980.

[16] K. Iwasaki, H. Kimura, S. Shimomura, and M. Yoshida. From Gauss to Painlevé, a Modern Theory of Special Functions, volume E 16. Aspects of Mathematics, 1991.

[17] M. Jimbo. Monodromy problem and the boundary condition for some Painlevé equations. Publ. Res. Inst. Math. Sci., 18:1137-1161, 1982. 
[18] M. Jimbo and T. Miwa. Monodromy preserving deformations of linear ordinary differential equations with rational coefficients II. Physica 2D, 2:no. 3, 407-448, 1981.

[19] M. Jimbo, T. Miwa, and K. Ueno. Monodromy preserving deformations of linear ordinary differential equations with rational coefficients I. Physica 2D, 2:no. 2, 306-352, 1981.

[20] E. R. Kolchin. Differential algebra and algebraic groups. Academic Press, New York, 1973. Pure and Applied Mathematics, Vol. 54.

[21] A.H.M. Levelt. Hypergeometric functions. Doctoral thesis, University of Amsterdam, 1961.

[22] S. Malek. A Fuchsian system with a reducible monodromy is meromorphically equivalent to a reducible Fuchsian system. Publication de L'Institut de Recherche Mathématique Avancée, 2000.

[23] S. Malek. On the reducibility of the Schlesinger equations. J. Dynam. Control Systems, 8:no. 4, 505-527, 2002.

[24] B. Malgrange. Sur les déformations isomonodromiques I. Singularités régulières, volume 37 of Mathematics and Physics. Progr. Math. Birkhäuser Boston, 1983.

[25] Yu.I. Manin. Frobenius manifolds, quantum cohomology and moduli spaces, volume 47. American Mathematical Society, 1999.

[26] J. Marsden and A. Weinstein. Reduction of symplectic manifolds with symmetry. Rep. Mathematical Phys., 5:no. 1, 121-130, 1974.

[27] M. Mazzocco. Picard and Chazy solutions to the PVI equation. Math. Ann., 321:no. 1, 131-169, 2001.

[28] M. Mazzocco. Rational solutions of the Painlevé VI equation. J. Phys. A: Math. Gen., 34:2281-2294, 2001

[29] M. Mazzocco. The geometry of the classical solutions of the Garnier systems. Int. Math. Res. Not., 2002:no.12, 613-646, 2002.

[30] T. Miwa. Painlevé property of monodromy presereving equations and the analyticity of $\tau$-functions. Publ. RIMS, 17:703-721, 1981.

[31] M. Ohtsuki. On the number of apparent singularities of a linear differential equation. Tokyo J. Math., 5:23-29, 1982.

[32] K. Okamoto. Isomonodromic deformations, Painlevé equations, and the Garnier system. J. Fac. Sci. Univ. Tokyo, Sect. 1A, Math., 33:576-618, 1986.

[33] K. Okamoto. Painlevé equations and Dynkin diagrams. Painlevé Transcendents, pages 299-313, 1992.

[34] K. Okamoto and H. Kimura. On Particular solutions of the Garnier system and the hypergeometric functions of several variables. Quart. J. Math. Oxford, 37:61-80, 1986.

[35] L. Schlesinger. Ueber eine Klasse von Differentsial System Beliebliger Ordnung mit Festen Kritischer Punkten. J. fur Math., 141:96-145, 1912.

[36] Y. Sibuya. Linear Differential Equations in the Complex Domain: Problems of Analytic Continuation, volume 82. AMS TMM, 1990.

[37] H. Umemura. Irreducebility of the first differential equation of Painlevé. Nagoya Math. J., 117:231-252, 1990.

[38] Marius van der Put and Michael F. Singer. Galois theory of linear differential equations, volume 328 of Grundlehren der Mathematischen Wissenschaften [Fundamental Principles of Mathematical Sciences]. Springer-Verlag, Berlin, 2003. 
Sissa, International School of Advanced Studies, via Beirut 2-4, 34014 Trieste, ITALY

E-mail address: dubrovin@sissa.it

School of Mathematics, The University of Manchester, Manchester M60 1QD, United Kingdom.

E-mail address: Marta.Mazzocco@manchester.ac.uk 